\title{
Plasma shaping and its impact on the pedestal of ASDEX Upgrade: Edge stability and inter-ELM dynamics at varied triangularity
}

\author{
F. M. Laggner ${ }^{1,2}$, E. Wolfrum ${ }^{3}$, M. Cavedon ${ }^{3}$, M. G. Dunne ${ }^{3}$, \\ G. Birkenmeier ${ }^{3,4}$, R. Fischer ${ }^{3}$, M. Willensdorfer ${ }^{3}$, F. Aumayr ${ }^{2}$, \\ the EUROfusion MST1 Team* and the ASDEX Upgrade Team \\ 1 Princeton University, Princeton, New Jersey 08544, United States of America \\ ${ }^{2}$ Institute of Applied Physics, TU Wien, Fusion@ÖAW, Wiedner Hauptstr. 8-10, \\ 1040 Vienna, Austria \\ ${ }^{3}$ Max Planck Institute for Plasma Physics, Boltzmannstr. 2, 85748 Garching, \\ Germany \\ 4 Physik-Department E28, Technische Universität München, James-Franck-Str.1, \\ 85748 Garching, Germany \\ * See author list of "H. Meyer et al. 2017 Nucl. Fusion 57 102014" \\ E-mail: flaggner@princeton.edu
}

\begin{abstract}
The plasma shape, in particular the triangularity $(\delta)$, impacts on the pedestal stability. A scan of $\delta$ including a variation of heating power $\left(P_{\text {heat }}\right)$ and gas puff was performed to study the behaviour of edge localised modes (ELMs) and the preELM pedestal stability for different plasma shapes. Generally, at higher $\delta$ the pedestal top electron density $\left(n_{\mathrm{e}}\right)$ is enhanced and the ELM repetition frequency $\left(f_{\mathrm{ELM}}\right)$ is reduced. For all $\delta$, the pedestal top $n_{\mathrm{e}}$ is already fully established to its pre-ELM value during the initial recovery phase of the $n_{\mathrm{e}}$ pedestal, which takes place immediately after the ELM crash. The lowering of the $f_{\mathrm{ELM}}$ with increasing $\delta$ is related to longer pedestal recovery phases, especially the last pre-ELM phase with clamped pedestal gradients (after the recovery phases of the $n_{\mathrm{e}}$ and electron temperature $\left(T_{\mathrm{e}}\right)$ pedestal) is extended. In all investigated discharge intervals, the pre-ELM pedestal profiles are in agreement with peeling-ballooning (PB) theory.

Over the investigated range of $\delta$, two well-separated $f_{\mathrm{ELM}}$ bands are observed in several discharge intervals. Their occurrence is linked to the inter-ELM pedestal stability. In both kinds of ELM cycles the pedestal evolves similarly, however, the 'fast' ELM cycle occurs before the global plasma stored energy ( $\left.W_{\mathrm{MHD}}\right)$ increases, which then provides a stabilising effect on the pedestal, extending the inter-ELM period in the case of the 'slow' ELM cycle. At the end of a 'fast' ELM cycle the $n_{\mathrm{e}}$ profile is radially shifted inwards relative to the $n_{\mathrm{e}}$ profile at the end of a 'slow' ELM cycle, leading to a reduced pressure gradient. The appearance of two $f_{\mathrm{ELM}}$ bands suggests that the pedestal becomes more likely PB unstable in certain phases of the inter-ELM evolution. Such a behaviour is possible because the evolution of the global plasma is not rigidly coupled to the evolution of the pedestal structure on the timescales of an ELM cycle.
\end{abstract}


PACS numbers: 52.55.-s, 52.55.Fa, 52.55.Tn

Keywords: Tokamak, H-mode, Edge localised modes, plasma shaping, ELM behaviour

Submitted to: Nucl. Fusion 


\section{Introduction}

In magnetically confined fusion plasmas the stability of the plasma edge, is of special importance. In the high confinement mode (H-mode), on the one hand high plasma performance can be achieved, on the other hand owing to the large pressure gradients at the edge, named the pedestal, instabilities can occur, so-called edge localised modes (ELMs). These cause large transient heat and particle fluxes towards the wall, which could exceed material limits in a future fusion device $[1,2,3]$. Hence, it will be necessary to keep the pedestal as high as possible (good plasma performance), and well below its stability limit (to avoid ELMs). To optimise pedestals in this sense, understanding of the mechanisms that determine the pedestal structure and its stability is required.

The presented work emphasises the impact of the plasma shape on the pedestal stability and its recovery in between ELM crashes in type-I ELMy H-mode plasmas. Under these plasma conditions it is known from magnetohydrodynamic (MHD) theory, that higher triangularity $(\delta)$ and plasma elongation $(\kappa)$ are beneficial for the plasma edge stability $[4,5,6]$, enabling steeper profile gradients and higher pedestal pressure. The positive impact of $\delta$ on the pedestal height has also been experimentally confirmed in several tokamak experiments $[7,8,9]$. It has been found that higher $\delta$ is connected to an increase of the electron density $\left(n_{\mathrm{e}}\right)$ pedestal $[10,11]$. However, this dependency could be also biased by changes of the strikepoint positions in the active divertor. These were necessary to vary $\delta$, however, such changes can impact on the neutral pumping efficiency and divertor conditions. For this reason, the presented experiments aimed for a variation of $\delta$ without changing the strike- and X-point positions in the active divertor.

Additionally to the changes in the pedestal structure, a variation of $\delta$ changes the ELM repetition frequency $\left(f_{\mathrm{ELM}}\right)$ [12]. In general, Type-I ELM behaviour, respectively $f_{\text {ELM }}$ and the duration of the ELM crash, can be influenced by several discharge conditions, e.g. impurity seeding $[13,14,15,16,17]$ or neutral influx like external gas puff $[18,19,20,21,22,23]$. A special change of ELM behaviour has been observed at ASDEX Upgrade, where two separated $f_{\text {ELM }}$ bands occurred [24, 25]. Similar observations have also been reported from the KSTAR experiment [26], here, referred to as secondary ELM peaks, and at TCV [27]. Also within the presented experiments, such an ELM behaviour was observed and the underlying mechanisms that cause two distinct $f_{\text {ELM }}$ bands are studied in this work.

In the following, the experimental procedure is introduced that was used to compare H-mode plasma discharges with a variation of $\delta$, heating power $\left(P_{\text {heat }}\right)$ and gas puff (section 2). In section 3 the general discharge behaviour is described, emphasising on the impact of $\delta$ on $f_{\mathrm{ELM}}, n_{\mathrm{e}}$ pedestal and plasma stored energy $\left(W_{\mathrm{MHD}}\right)$. The stabilising effect of $\delta$ on the pedestal is discussed in section 4 and the inter-ELM evolution for two cases with low and high $\delta$ are compared. The occurrence of two distinct $f_{\text {ELM }}$ bands is analysed at low and high $\delta$ in section 5 . In section 6 the main results are recapped, showing that the increased pedestal top $n_{\mathrm{e}}$ at enlarged $\delta$ is already established in the initial inter-ELM recovery phase of the pedestal. At higher $\delta, f_{\mathrm{ELM}}$ decreases because the 
inter-ELM recovery phases are prolonged, especially the pre-ELM phase with clamped pedestal pressure gradients [28] is extended. When two distinct $f_{\text {ELM }}$ bands are observed, both types of ELM cycles show a similar pedestal evolution up to the point where the 'fast' ELM cycle (higher $f_{\text {ELM }}$ band) ends. From this point on $W_{\text {MHD }}$ increases providing via the Shafranov shift a stabilising effect on the pedestal and extending the 'slow' ELM cycle (lower $f_{\text {ELM }}$ band).

\section{Conducted experiment}

To perform a $\delta$ scan without changing the divertor conditions in the active divertor, a lower single null (LSN) plasma scenario was chosen and only the upper triangularity $\left(\delta_{\mathrm{up}}\right)$ was varied. The achievable range of $\delta$ is limited by operational boundaries on maximum shaping coil currents in the ASDEX Upgrade standard scenario [29] with 1.0 MA plasma current $\left(I_{\mathrm{p}}\right)$ and $-2.5 \mathrm{~T}$ toroidal magnetic field $\left(B_{\mathrm{t}}\right)$. The negative sign of $B_{\mathrm{t}}$ stands for its opposite direction to $I_{\mathrm{p}}$. Further, increasing $\delta$ increases the $n_{\mathrm{e}}$ pedestal, which can lead to $n_{\mathrm{e}}$ above the electron cyclotron emission (ECE) cut-off and therefore, limiting the diagnostic capabilities. For these reasons, a plasma scenario with reduced $I_{\mathrm{p}}$ in contrast to the ASDEX Upgrade standard configuration was chosen. The main parameters were $I_{\mathrm{p}}=0.8 \mathrm{MA}$ and $B_{\mathrm{t}}=-2.5 \mathrm{~T}$. This gives a higher safety factor $(q)$ at the edge in comparison to the standard configuration, lowers the required coil currents for shaping by roughly $20 \%$, lowers the central plasma $n_{\mathrm{e}}$ and extends the achievable discharge length because of less flux consumption of the transformer coil.

\subsection{Shape comparison and varied parameters}

Usually, changing the shaping coil currents impacts $\delta$ and $\kappa$ when the outer strikepoint location is controlled. Since $\kappa$ also influences the plasma stability, it was emphasised to adjust the shaping accordingly that only minor variations of $\kappa$ in the region of $\pm 5 \%$ occur. Further, changes of $P_{\text {heat }}$ and correspondingly $W_{\text {MHD }}$ impact on the Shafranov shift and therefore, modify the plasma shape. To avoid these modifications, the shaping coil currents were adjusted, such that $\delta$ and $\kappa$ remained unchanged, when steps in $P_{\text {heat }}$ were performed.

The general experimental procedure was to establish a plasma scenario at fixed $\delta$ and then vary $P_{\text {heat }}$ and neutral gas puff. Then, $\delta$ was varied from discharge to discharge. By this procedure the achieved range of the upper triangularity $\left(\delta_{\text {up }}\right)$ was 0.0 to 0.4 leading to an average $\delta$ between 0.21 and 0.41 .

Figure 1 presents a comparison of the plasma shapes for low (dark blue), medium (dark red) and high (orange) $\delta$.

At low and medium delta a nice match of X-point and strikepoint locations were achieved, while the location of the plasma top was moved inwards resulting in an increased $\delta$. For the high $\delta$ scenario a small change in the strikeline positions of the order of $3 \mathrm{~cm}$ had to be accepted. Since the targets in this region are usually well conditioned 


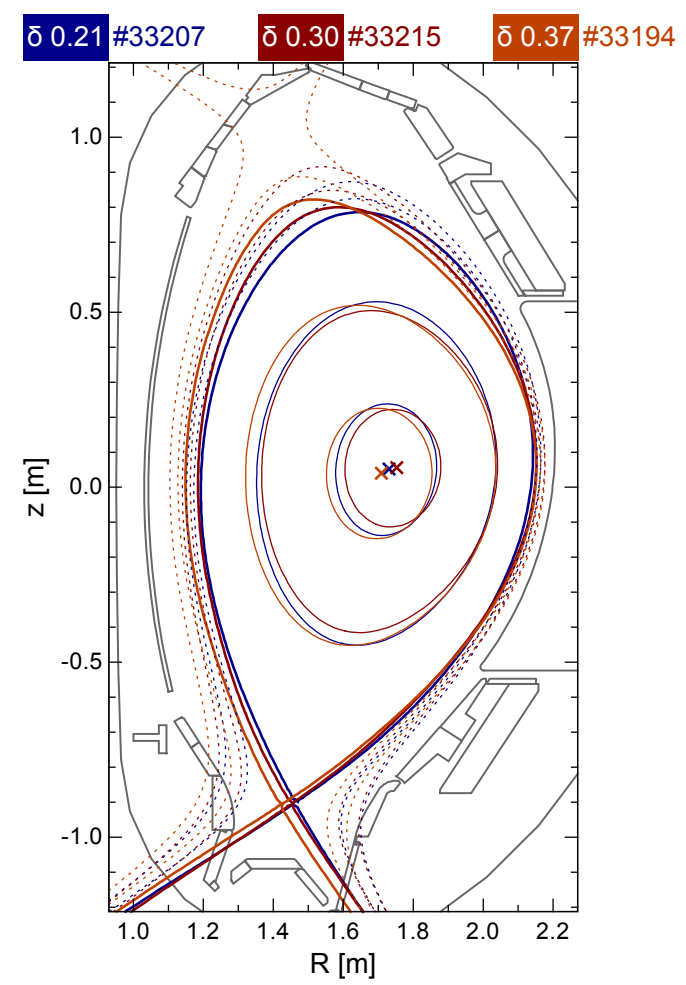

Figure 1: Comparison of separatrix locations with varied $\delta_{\text {up }}$ : At low (0.21, dark blue) and medium (0.30, dark red) $\delta$ the strike- and X-point locations are the same, whereas at high $(0.37$, orange) $\delta$ the strikelines have slightly different locations (shift at the outer target in the region of $3 \mathrm{~cm})$ and the lower triangularity $\left(\delta_{\text {low }}\right)$ is higher.

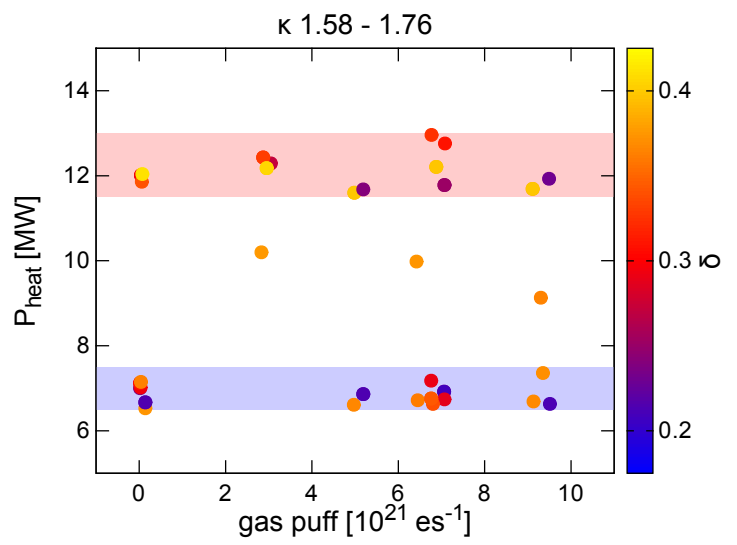

Figure 2: Varied control parameters: At mainly two levels of $P_{\text {heat }}$ (6.5 MW to 7.5 MW, blue shaded area and 11.5 MW to $13.0 \mathrm{MW}$, red shaded area) a scan of the gas puff was performed for the different $\delta$. A variation of $\kappa$ in the region of $\pm 5 \%$ was tolerated.

no impact on the divertor conditions or neutral recycling is expected by this change.

The accessed parameter range of $P_{\text {heat }}$ and neutral gas puff is presented in figure 2 . In principle two levels of $P_{\text {heat }}$ were applied: A lower level between $6.5 \mathrm{MW}$ and $7.5 \mathrm{MW}$ 
(blue shaded area) and a higher level in the range from 11.5 MW to 13.0 MW (red shaded area). The gas puff was varied between $0 \cdot 10^{21} \mathrm{es}^{-1}$ and $10 \cdot 10^{21} \mathrm{es}^{-1}$ in such a way that it was stepwise reduced throughout and turned off completely towards the end of the discharge.

\subsection{Performed data analysis}

This study is mainly focused on the investigation of the electron profile structures and especially their inter-ELM dynamics. The $n_{\mathrm{e}}$ and electron temperature $\left(T_{\mathrm{e}}\right)$ profiles are evaluated using the integrated data analysis (IDA) approach [30], which combines and evaluates the data of multiple plasma diagnostics. For the presented profiles, the following diagnostics were included in the analysis: The deuterium cyanide laser interferometry diagnostic [31] for the core $n_{\mathrm{e}}$, the lithium beam diagnostic [32] for the edge $n_{\mathrm{e}}$ and the recently refurbished ECE diagnostic [33] together with an electron cyclotron forward model [34] for the $T_{\mathrm{e}}$. The relative alignment of the $n_{\mathrm{e}}$ and $T_{\mathrm{e}}$ profiles was determined by cross comparison to the corresponding profiles of the Thomson scattering (TS) diagnostic [35]. If necessary, the $T_{\mathrm{e}}$ profile is slightly shifted $(<3 \mathrm{~mm})$ such that $100 \mathrm{eV}$ are the separatrix $T_{\mathrm{e}}$ [36]. This minor shift is then also applied to the $n_{\mathrm{e}}$ profile. The quality of the raw data allows a profile evaluation with a temporal resolution of $250 \mu \mathrm{s}$.

To reconstruct the plasma equilibria and corresponding quantities, e.g. $\delta$ and $\kappa$, the integrated data analysis equilibrium (IDE) solver [37] was routinely applied, which implements a constraint on the pedestal pressure profile according to the one evaluated by IDA at the corresponding time. The equilibria are reconstructed at a temporal resolution of $1 \mathrm{~ms}$. Within the presented work, for the first time these 'IDE equilibria' have routinely been used to serve as input for linear MHD stability, peeling-ballooning (PB), analyses. Furthermore, an ELM synchronisation technique was implemented to statistically average over multiple equilibria with respect to the corresponding ELM onsets. This allows to determine the uncertainties in average toroidal current density in the pedestal $\left(\left\langle j_{\text {tor }}\right\rangle\right)$ and normalised pressure gradient $(\alpha)$ and furthermore, to display error bars on the operational point. The resulting stability diagrams for low and high $\delta$ cases as well as the different types of ELM cycles are are presented in the sections 4.1 and 5.3.

\section{General observations}

In the following, the overall discharge behaviour at different $\delta$ with respect to the pedestal and ELM behaviour is discussed and compared to previous experimental results. As shown in figure $3, f_{\text {ELM }}$ decreases at higher $\delta$, which is in agreement with previous observations [12]. In this figure only discharge intervals with a single $f_{\text {ELM }}$ band are considered. The vertical bars represent the scattering of $f_{\mathrm{ELM}}$. This mainly appears due to varying duration of the last, pre-ELM phase of the inter-ELM pedestal evolution for 


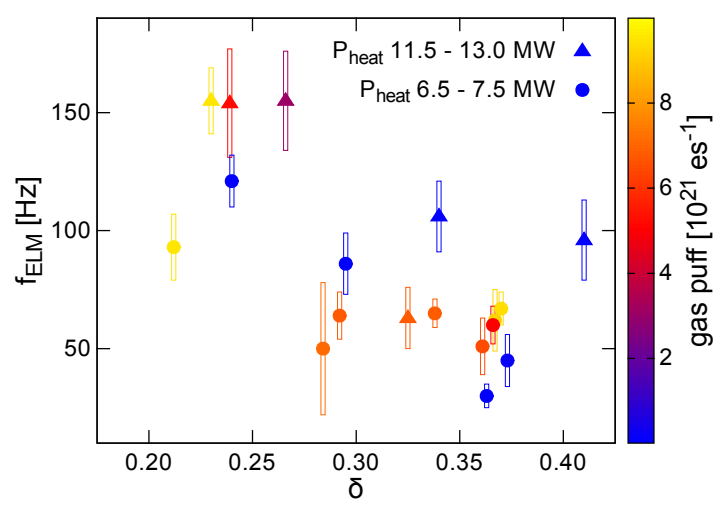

Figure 3: Dependency of $f_{\mathrm{ELM}}$ on $\delta$ : Only discharge intervals with a single $f_{\mathrm{ELM}}$ are considered and the vertical bars correspond to the statistical scattering of $f_{\text {ELM }}$. For high (triangles) and low (circles) $P_{\text {heat }}, f_{\text {ELM }}$ decreases with $\delta$. Additional scattering in the data might be due to the varying gas puff.
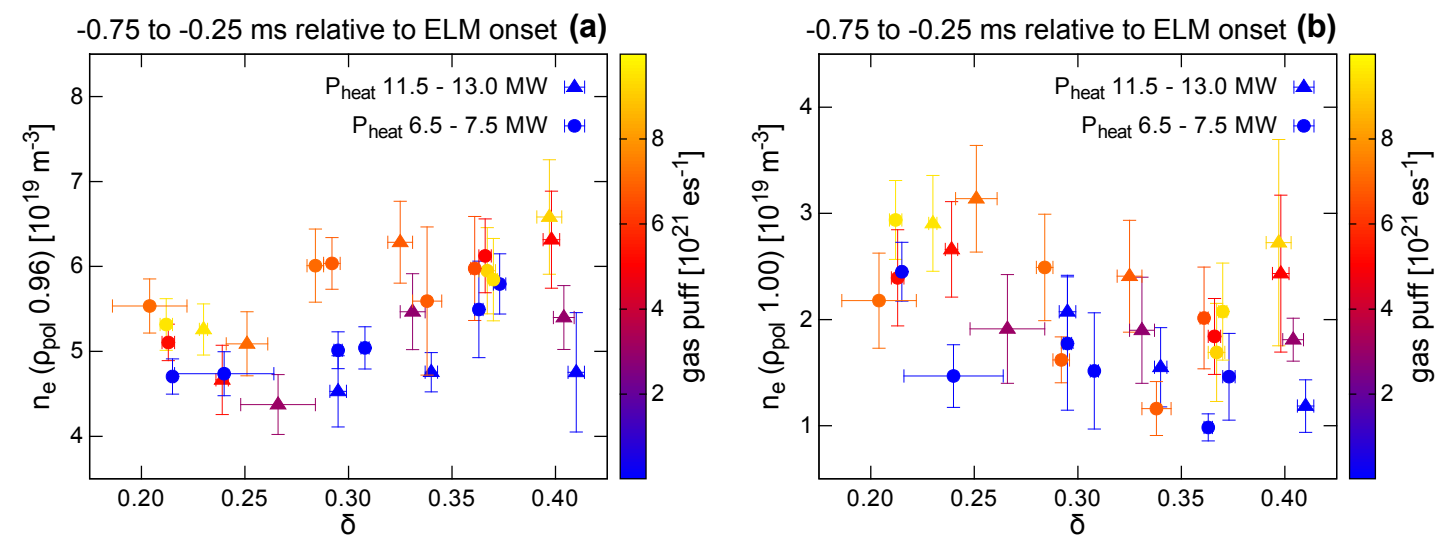

Figure 4: Impact of $\delta$ on the $n_{\mathrm{e}}$ pedestal: (a) the pedestal top $n_{\mathrm{e}}\left(\rho_{\mathrm{pol}}=0.96\right)$ and (b) the separatrix $n_{\mathrm{e}}\left(\rho_{\mathrm{pol}}=1.00\right)$. The pre-ELM pedestal top $n_{\mathrm{e}}$ increases with $\delta$, whereas the separatrix $n_{\mathrm{e}}$ is reduced, indicating a steepening of the pedestal $\nabla n_{\mathrm{e}}$.

the individual ELM cycles. In comparison to the lower level $P_{\text {heat }}$ (points) $f_{\mathrm{ELM}}$ increases at the higher level of $P_{\text {heat }}$ (triangles), which is characteristic for type-I ELMs. For both levels of $P_{\text {heat }}$ the anti-correlation of $f_{\mathrm{ELM}}$ and $\delta$ is observed. Scattering of the data could be related to the applied gas puff, which also impacts on $f_{\mathrm{ELM}}$, however, no clear ordering in terms of gas puff can be seen (compare colour scale). In this comparison it is evident, that the impact of $\delta$ on $f_{\mathrm{ELM}}$ is much stronger than the one of the gas puff in the investigated parameter range.

Another general trend of previous studies is that $n_{\mathrm{e}}$ increases with $\delta[10,38]$. This is also reproduced in the conducted experiment at higher gas puff as shown in figure $4 \mathrm{a}$. Here, the pre-ELM pedestal top $n_{\mathrm{e}}$, averaged between $-0.75 \mathrm{~ms}$ and $-0.25 \mathrm{~ms}$ relative to the ELM onset and measured at $\rho_{\text {pol }}=0.96$, is plotted in dependency of $\delta$. At 

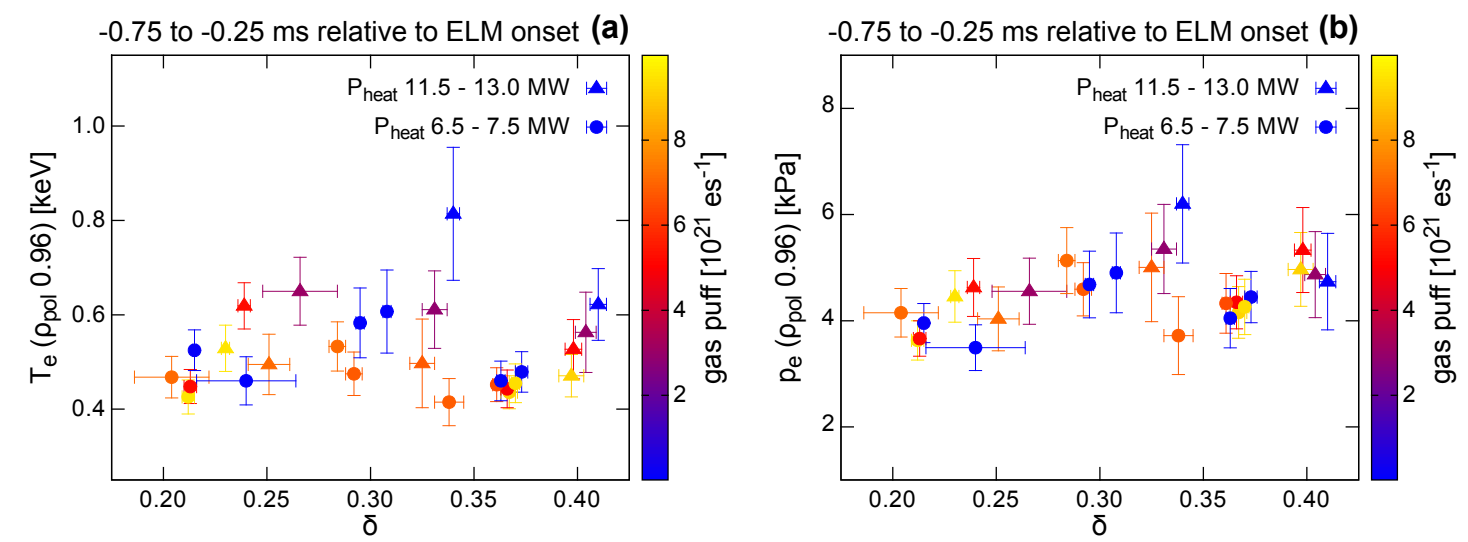

Figure 5: Pedestal temperature and pressure in dependence of $\delta:$ (a) the pedestal top $T_{\mathrm{e}}$ and (b) pedestal top $p_{\mathrm{e}}\left(\rho_{\mathrm{pol}}=0.96\right)$. The pedestal top $T_{\mathrm{e}}$ shows in average no clear trend for varying $\delta$ but an anti-correlation with the gas puff (colour scale) can be seen. In combination with the increasing pedestal top $n_{\mathrm{e}}$ the pedestal top $p_{\mathrm{e}}$ increases for higher $\delta$. At fixed $\delta$ the pedestal top $p_{\mathrm{e}}$ is independent of the gas puff.

medium and high gas puff (orange and yellow markers) the trend is visible. Whereas, at low gas puff and high $P_{\text {heat }}$ (blue and purple triangles) significantly lower pedestal top $n_{\mathrm{e}}$ are measured at very large $\delta(>0.40)$ than expected from the trend at lower $\delta$. No clear dependence of the pedestal top $n_{\mathrm{e}}$ on the two different $P_{\text {heat }}$ (compare points and triangles) can be identified.

The other important contribution to the $n_{\mathrm{e}}$ pedestal structure is the separatrix $n_{\mathrm{e}}$, which in a simplified approximation together with the pedestal top $n_{\mathrm{e}}$ sets the pedestal $\nabla n_{\mathrm{e}}$ (at unchanged pedestal width). The separatrix $n_{\mathrm{e}}$ is presented in figure $4 \mathrm{~b}$ and exhibits, as indicated in previous studies [23], a strong dependency on the gas puff. Further, it decreases towards higher $\delta$, especially at low gas puffs (blue and purple markers). Again the discharge intervals for $\delta>0.40$ mark the exception to the trend, this time in the medium to high gas puff range. Since no obvious reason could be identified for this deviation, the intervals were not excluded from the following analysis. It remains unclear if the observed deviations for the few cases with $\delta>0.40$ are actually related to $\delta$ or simply due to individual discharge conditions. In principle the separatrix $n_{\mathrm{e}}$ is not rigidly coupled to the gas puff, rather, it is impacted by the divertor and scrape-off layer (SOL) conditions, which themselves are dependent on the gas puff. It seems reasonable that when varying $\delta$ (at constant gas puff), the SOL conditions are affected and therefore, the separatrix $n_{\mathrm{e}}$ could be varied without a change of the gas puff.

The combination of the increasing pedestal top $n_{\mathrm{e}}$ with the pre-ELM $T_{\mathrm{e}}$ pedestal, forming the electron pressure $\left(p_{\mathrm{e}}\right)$ pedestal is presented in figure 5 . In average the 'pedestal top' $T_{\mathrm{e}}$, determined at $\rho_{\mathrm{pol}}=0.96$, does not show a clear dependence on $\delta$ and is in the region of $400 \mathrm{eV}$ to $550 \mathrm{eV}$ at low $P_{\text {heat }}$ and between $500 \mathrm{eV}$ and $650 \mathrm{eV}$ at high $P_{\text {heat }}$ (except for one outlier). The anti-correlation of the pedestal top $T_{\mathrm{e}}$ with the gas 

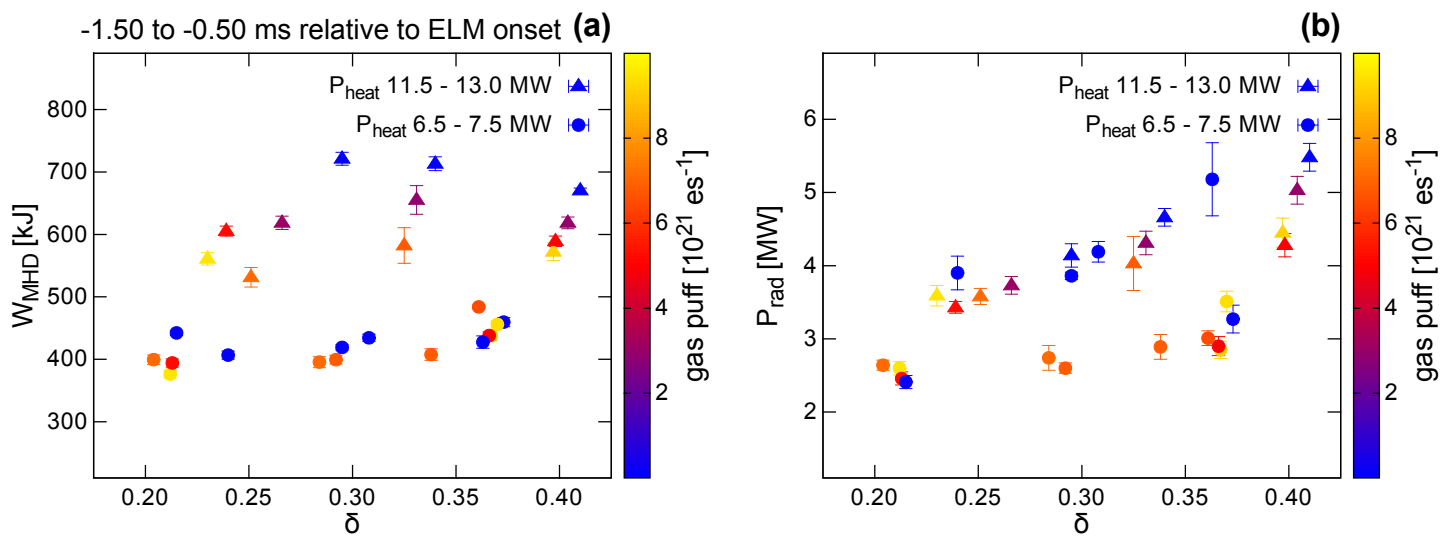

Figure 6: Global plasma properties in relation to $\delta$ : (a) $W_{\text {MHD }}$ and (b) $P_{\text {rad }}$. As expected a separation of $W_{\text {MHD }}$ for the different $P_{\text {heat }}$ is observed. At high $P_{\text {heat }}$ a clear degradation of $W_{\text {MHD }}$ can bee seen with increased gas puff (colour scale).

puff can be seen comparing the colour scaling. This can be related to the correlation of the gas puff and the pedestal top $n_{\mathrm{e}}$, under the condition that the pedestal pressure profile is fixed for a given shape. The pedestal top pressure are compared in figure $5 \mathrm{~b}$. Up to a $\delta$ of 0.34 an increase in the pedestal $p_{\mathrm{e}}$ might be seen, which would be consistent with previous results $[8,9]$. However, over the full investigated range of $\delta$ no clear trend of $p_{\mathrm{e}}$ can be identified.

Further, the global $W_{\text {MHD }}$ does not show any strong dependency on $\delta$ as presented in figure 6 a. The most dominant impact on $W_{\text {MHD }}$ is given by $P_{\text {heat }}$, when comparing points and triangles. By applying approximately two times the $P_{\text {heat }}, W_{\text {MHD }}$ is increased by roughly $50 \%$ in the covered $\delta$ range. At the higher level of $P_{\text {heat }}$ a separation for the different applied gas puffs can be seen (colour scale). Here, the largest $W_{\text {MHD }}$ is achieved at low gas puff. Previously, it has been reported that high gas puff negatively impact on global confinement in H-mode via degradation of the pedestal top pressure $[39,40]$. However, this is not the case here, since the pedestal top $p_{\mathrm{e}}$ do not exhibit a clear connection to the gas puff.

The weak relation between pedestal top $p_{\mathrm{e}}$ and $W_{\mathrm{MHD}}$ (compare figures $5 \mathrm{~b}$ and $6 \mathrm{a}$ ) indicate that the pedestal and the core plasma are not rigidly coupled meaning that additional power losses occur from the core region at higher $\delta$. A possible candidate for such a loss is radiation and especially with the tungsten (W) wall at ASDEX Upgrade when reducing the gas puff, impurity accumulation and enlarged radiation losses occur $[41,42]$. For this reason the averaged $P_{\text {rad }}$ are plotted in figure $6 \mathrm{~b}$ for the investigated cases. At constant $P_{\text {heat }}$ a clear increase of $P_{\text {rad }}$ towards higher $\delta$ is observed, which was also seen on JET [43]. Only at selected cases of low $P_{\text {heat }}$ and low gas puff (blue circles), significantly higher $P_{\text {rad }}$ occurs than at higher gas puffs, similar shape and similar $P_{\text {heat }}$. For these cases of enhanced $P_{\text {rad }}$ also an increased $\mathrm{W}$ concentration is monitored and the estimated radiation increase due to $\mathrm{W}$ is consistent with the measured $P_{\text {rad }}$ increase. The overall increase of $P_{\text {rad }}$ with $\delta$ can not be associated to enhanced $\mathrm{W}$ concentrations. It is 

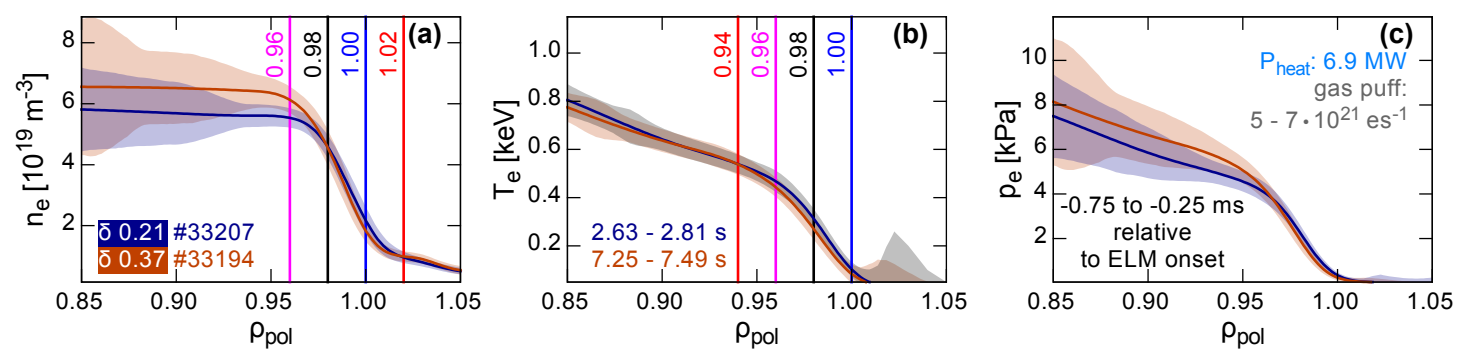

Figure 7: Comparison of the pre-ELM pedestal structure at low (0.21, dark blue) and high (0.37, orange) $\delta$ : (a) $n_{\mathrm{e}}$, (b) $T_{\mathrm{e}}$ and (c) $p_{\mathrm{e}}$ profile. At similar gas puff and $P_{\text {heat }}$ the $n_{\mathrm{e}}$ pedestal increases with $\delta$, while $T_{\mathrm{e}}$ pedestal remains similar. In combination, a higher and steeper $p_{\mathrm{e}}$ pedestal is found at higher $\delta$.

more likely that the increased radiation losses at high $\delta$ are caused by the combination of a larger plasma volume and a higher averaged plasma density.

The enhanced radiation can partially explain the weaker coupling between the pedestal top $p_{\mathrm{e}}$ and $W_{\mathrm{MHD}}$, however, it can not be the reason for the anti-correlation of $W_{\text {MHD }}$ and gas puff at high $P_{\text {heat }}$, since $P_{\text {rad }}$ also tends to increase at reduced gas puff. Possible reasons that could explain the degradation of $W_{\text {MHD }}$ with gas puff at high $P_{\text {heat }}$ are outward shifted deposition profiles of the neutral beam heating power $\left(P_{\mathrm{NBI}}\right)$ (because higher gas puff increases the density) or changes in core transport and MHD behaviour.

\section{Impact of triangularity on the pedestal}

As already discussed in section 3 the pedestal top $n_{\mathrm{e}}$ increases at higher $\delta$. In the following the relation between pedestal structure and $\delta$ is analysed and compared to $\mathrm{PB}$ stability analysis.

\subsection{Pedestal structure and stability}

To investigate the impact of $\delta$ on the pedestal structure and stability, two different discharge intervals, performed at the lower level of $P_{\text {heat }}$, are investigated. These have a similar $\kappa$ and the applied gas puff only varies between $5 \cdot 10^{21} \mathrm{es}^{-1}$ and $7 \cdot 10^{21} \mathrm{es}^{-1}$. The corresponding pre-ELM profiles of $n_{\mathrm{e}}, T_{\mathrm{e}}$ and $p_{\mathrm{e}}$ are compared in figure 7 . The two different discharge intervals correspond to the low (0.21, dark blue) and high $(0.37$, orange) $\delta$. The vertical lines correspond to different radial locations at which layers of $n_{\mathrm{e}}$ and $T_{\mathrm{e}}$ are tracked throughout the ELM cycle in figure 9.

At high $\delta$ the pedestal top $n_{\mathrm{e}}$ increases (see figure $7 \mathrm{a}$ ) and the $T_{\mathrm{e}}$ profiles (figure $7 \mathrm{~b}$ ) of the low and high $\delta$ case are similarly shaped. Maybe at high $\delta$, the $T_{\mathrm{e}}$ profile is slightly shifted inwards, however, this is within the experimental uncertainties. The combined $p_{\mathrm{e}}$ profiles (figure $7 \mathrm{c}$ ) reflect the variation of $n_{\mathrm{e}}$. At higher $\delta$ a higher $p_{\mathrm{e}}$ pedestal is observed, owing to the change of the $n_{\mathrm{e}}$ profile structures in combination with similar 


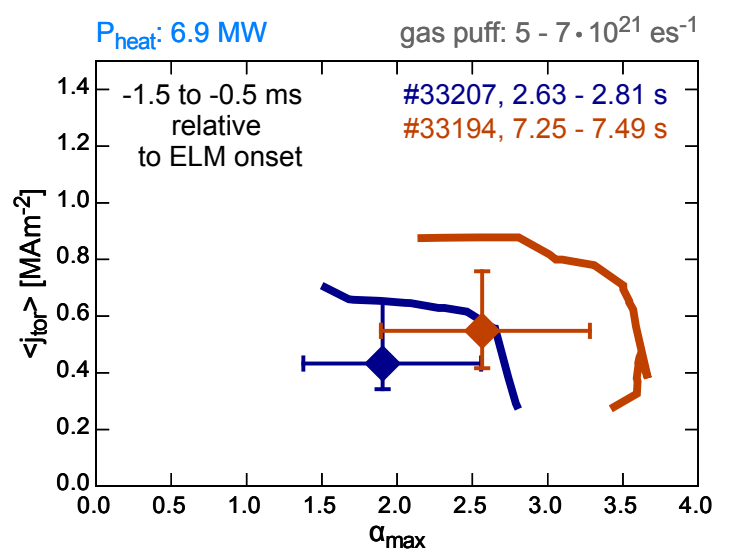

Figure 8: PB stability diagram for low (dark blue) and high (orange) $\delta$ : The stability boundary at high $\delta$ is shifted towards higher $\alpha_{\max }$ and $\left\langle j_{\text {tor }}\right\rangle$. The steeper $p_{\mathrm{e}}$ gradient at high $\delta$ moves the operational point towards larger $\alpha_{\max }$.

$T_{\mathrm{e}}$ profiles. For the selected discharge intervals of this comparison, $W_{\mathrm{MHD}}$ at high $\delta$ is also higher according to the increased $p_{\mathrm{e}}$ pedestal.

As theoretically predicted, the steeper pressure gradients are achievable owing to the stabilising effect of higher $\delta[44,45]$. For the low and high $\delta$ pre-ELM equilibria, linear MHD stability analyses were performed using the stability workflow at ASDEX Upgrade [46]. Here, the ELM synchronised equilibria reconstructed with the IDE solver were used as it was introduced in section 2.2. The corresponding operational points and stability boundaries are represented in quantities of $\left\langle j_{\text {tor }}\right\rangle$ and maximum normalised pressure gradient $\left(\alpha_{\max }\right)$. The $\mathrm{j}$ - $\alpha$ diagram for the low and high $\delta$ case are presented in figure 8. At higher $\delta$ the $\mathrm{PB}$ boundary is extended towards larger $\left\langle j_{\text {tor }}\right\rangle$ and $\alpha_{\max }$. This allows the operational point to shift towards larger $\alpha_{\max }$ as also shown in figure $7 \mathrm{c}$. The investigated pre-ELM pedestals are in agreement with PB theory. At high $\delta$ the distance between the operational point and the PB boundary increases in comparison to the low $\delta$ case, however, this is not a robust observation and usually within the uncertainties in the locations of the operational point and the PB boundary. The uncertainties of the operational point are represented by the standard deviations from the averaged pre-ELM equilibria.

\subsection{Inter-ELM evolution}

The comparison of the inter-ELM pedestal development for the different $\delta$ can give insight into the changes of $f_{\mathrm{ELM}}$ and, therefore, the temporal approach of the stability limit. Further, the profile dynamics in between ELMs can be used to test models of the pedestal, as recently done for JET discharges [47]. Figure 9 presents the dynamics of the pedestal $n_{\mathrm{e}}$ and $T_{\mathrm{e}}$ profiles throughout the ELM cycle, in discharge intervals with single $f_{\text {ELM }}$ bands, at low (figures $9 \mathrm{a}$ and $9 \mathrm{~b}$ ) and high (figures $9 \mathrm{c}$ and $9 \mathrm{~d}$ ) $\delta$. The $n_{\mathrm{e}}$ profiles are tracked on four radial locations from the SOL $\left(\rho_{\mathrm{pol}}=1.02\right)$ towards the 

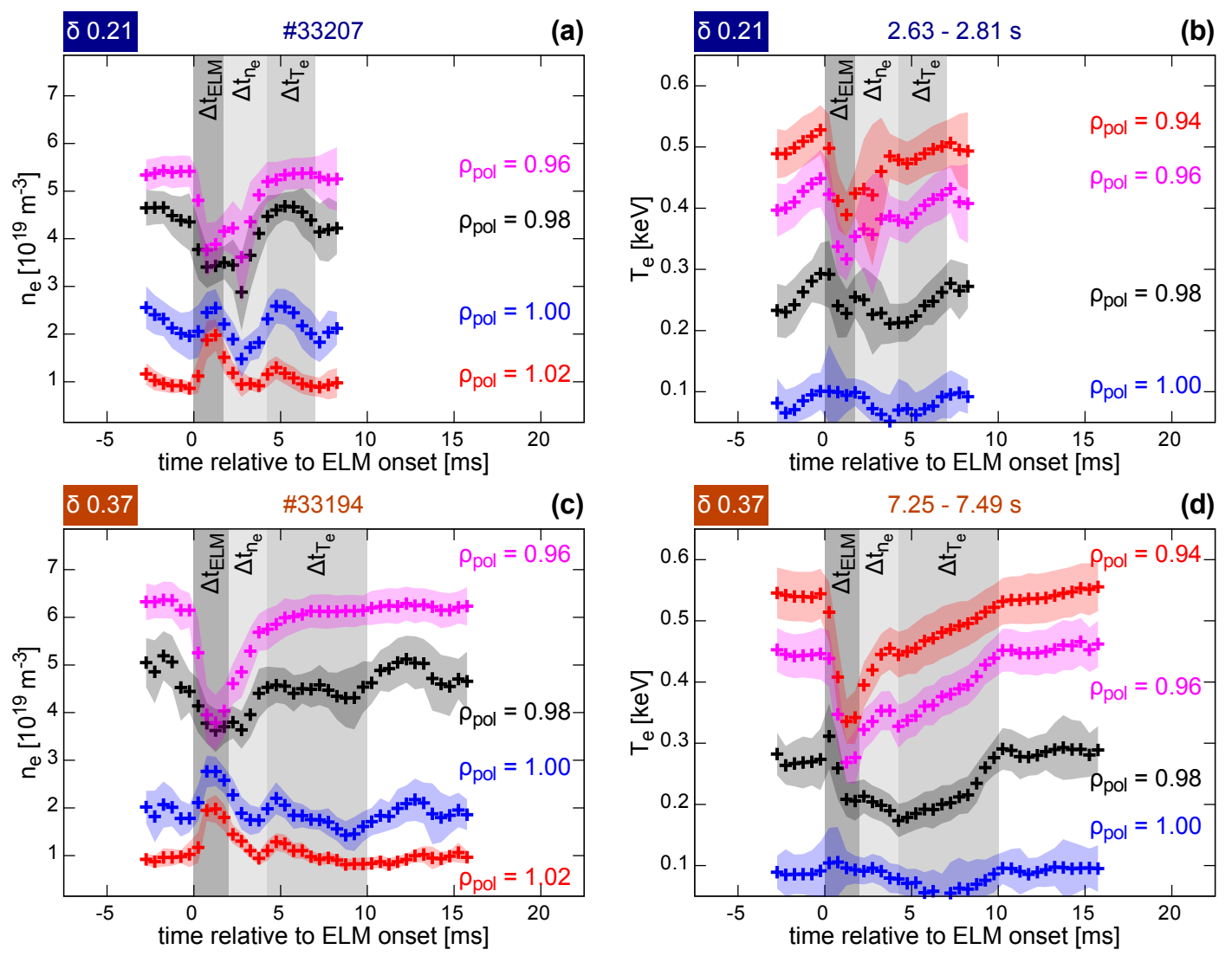

Figure 9: Inter-ELM pedestal evolution: $(\mathrm{a}, \mathrm{c}) n_{\mathrm{e}}$ and $(\mathrm{b}, \mathrm{d}) T_{\mathrm{e}}$ at certain radial positions across the pedestal $\left(\rho_{\mathrm{pol}}\right)$ for $(\mathrm{a}, \mathrm{b})$ low and $(\mathrm{c}, \mathrm{d})$ high $\delta$. For all cases the $n_{\mathrm{e}}$ pedestal is re-established before the $T_{\mathrm{e}}$ pedestal and the sequence of recovery phases is similar. The $T_{\mathrm{e}}$ pedestal recovery phase $\left(\Delta t_{T_{\mathrm{e}}}\right)$ and the pre-ELM phase increase their duration with increasing $\delta$, which corresponds to a decrease of $f_{\mathrm{ELM}}$.

pedestal top $\left(\rho_{\mathrm{pol}}=0.96\right)$ and the $T_{\mathrm{e}}$ profiles are tracked in the confined plasma region from $\rho_{\text {pol }}=1.00$ to $\rho_{\text {pol }}=0.94$ (see vertical lines in figure 7 ).

At all $\delta$ the sequence of pedestal recovery phases is similar to the one typical for ASDEX Upgrade, which was previously observed in experiments with a pedestal collisionality variation [28] and in isotope comparison studies [48]. After the ELM crash with duration $\Delta t_{\mathrm{ELM}}$, the $n_{\mathrm{e}}$ pedestal recovers first $\left(\Delta t_{n_{\mathrm{e}}}\right)$, followed by the recovery of the $T_{\mathrm{e}}$ pedestal $\left(\Delta t_{T_{\mathrm{e}}}\right)$ and the pre-ELM phase in which maximum electron pressure gradient $\left(\max \left(-\nabla p_{\mathrm{e}}\right)\right)$ is clamped [24]. In this phase also high frequency magnetic fluctuations set in, which are associated with an instability with toroidal mode numbers $(n)$ in the region of 10 [49].

The inter-ELM recovery rates can give information on the particle and heat fluxes towards the pedestal. For the $n_{\mathrm{e}}$ pedestal the recovery times are too short to perform a quantitative comparison of electron density recovery rate $\left(\partial n_{\mathrm{e}} / \partial t\right)$ for the different $\delta$ cases. Nevertheless, it is remarkable that the higher pedestal top $n_{\mathrm{e}}$ at high $\delta$ (see figure 4 a) is already established in this early phase of the ELM cycle. This suggest that 
the $n_{\mathrm{e}}$ pedestal is set first, while the pedestal top $T_{\mathrm{e}}$ is then determined by the maximum achievable pedestal $p_{\mathrm{e}}$, which is $\mathrm{PB}$ limited. The duration of the $T_{\mathrm{e}}$ pedestal recovery phase, $\Delta t_{T_{\mathrm{e}}}$, prolongs at higher $\delta$. Nevertheless, the $T_{\mathrm{e}}$ pedestal (positions $\rho_{\text {pol }}=0.96$ and $\left.\rho_{\text {pol }}=0.94\right)$ recovers at similar electron temperature recovery rate $\left(\partial T_{\mathrm{e}} / \partial t\right)$ during $\Delta t_{T_{\mathrm{e}}}$ for both $\delta$ cases, which suggests similar power fluxes to the pedestal. The longer $\Delta t_{T_{\mathrm{e}}}$ in the high $\delta$ case is caused by higher $W_{\mathrm{MHD}}$ losses per ELM, which leads to a larger drop of the $T_{\mathrm{e}}$ pedestal.

Most prominently, the duration of the pre-ELM phase with clamped pedestal pressure gradient almost doubles from low to high $\delta$. This is the largest contribution to the observed lower $f_{\text {ELM }}$ at higher $\delta$ and indicates that at high $\delta$ especially this period is more stable and therefore, prolonged.

In summary, the higher pedestal top $n_{\mathrm{e}}$ at high $\delta$ is found to be established immediately after the ELM crash in the $n_{\mathrm{e}}$ pedestal recovery phase. The phase of the $T_{\mathrm{e}}$ recovery in the ELM cycle is longer at higher $\delta$ and especially the pre-ELM phase with clamped electron pressure gradient $\left(\nabla p_{\mathrm{e}}\right)$. For the $T_{\mathrm{e}}$ pedestal recovery phase this prolongation is caused by higher ELM losses. The extension of the pre-ELM phase might be linked either to enhanced particle and heat fluxes across the pedestal than at low $\delta$, which saturate the stable pedestal structure longer and more reliably. Or at high $\delta$, the pedestal is more stable due to shaping in the sense that small pressure perturbations, which would already cause ELM crashes at low $\delta$, are stabilised and the pre-ELM phase is therefore extended.

\section{ELM frequency bands}

At all investigated $\delta$, discharge phases with two $f_{\text {ELM }}$ bands were observed. Their appearance cannot be related to certain parameter combinations of $P_{\text {heat }}$ and gas puff. For each $\delta$ it is rather set by an individual parameter combination. At high $\delta$, it is more likely that the two $f_{\text {ELM }}$ bands appear at the higher level of $P_{\text {heat }}$.

\subsection{Observation of 'slow' and 'fast' ELM cycles}

The two $f_{\text {ELM }}$ bands are associated to ELM cycles of different duration. Therefore, according to the observation, it is discriminated between 'fast' and 'slow' ELM cycles, which are related to the high and low $f_{\text {ELM }}$ band. Exemplary time traces of discharge intervals, which contain two $f_{\mathrm{ELM}}$ bands, are displayed in figure 10 a at low $\delta$ and in figure $10 \mathrm{~b}$ at high $\delta$. Here, $W_{\text {MHD }}$, pedestal top $p_{\mathrm{e}}$ (tracked at $\left.\rho_{\text {pol }}=0.96\right), f_{\text {ELM }}$ and inner divertor current are shown. The burst in the divertor current is associated to the ELM crash, $f_{\mathrm{ELM}}$ is determined by the inversion of the time period from an ELM crash until the following one. A similar $P_{\text {heat }}$ is applied in both discharge intervals. In agreement with the data presented in figure 3 , both $f_{\mathrm{ELM}}$ bands have a higher frequency at low $\delta$. The frequencies, which discriminate the $f_{\mathrm{ELM}}$ bands, are indicated by the horizontal, red dashed lines. The higher frequency band, the 'fast' ELM cycles, have blue 


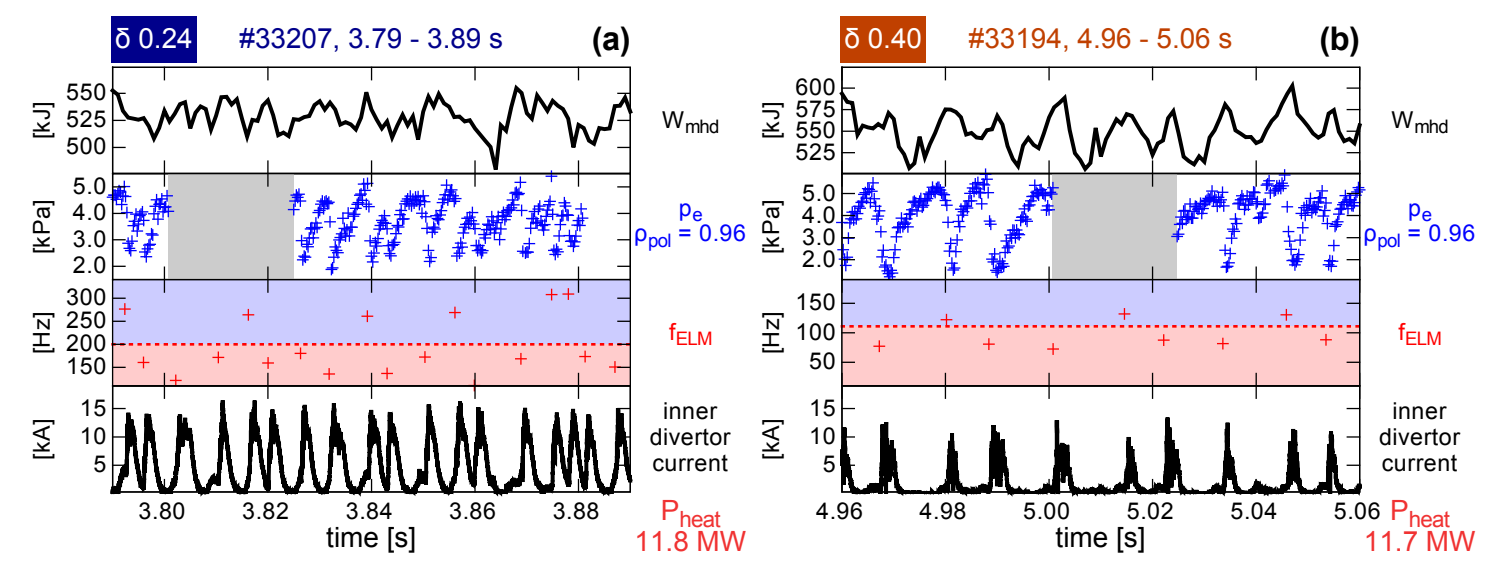

Figure 10: Discharge intervals with two $f_{\text {ELM }}$ bands: Time traces of $W_{\text {MHD }}$, pedestal top $p_{\mathrm{e}}, f_{\mathrm{ELM}}$ and inner divertor current at (a) low and (b) high $\delta$. The threshold in $f_{\text {ELM }}$ discriminating between 'fast' (blue background) and 'slow' (red background) ELM cycles is indicated by the red dashed line. Both types of ELM cycles appear irregularly.

\begin{tabular}{c|c|c|c|c|c|c}
\hline discharge & $\delta$ & $P_{\text {heat }}[\mathrm{MW}]$ & ELM cycle & $f_{\mathrm{ELM}}[\mathrm{Hz}]$ & $\Delta t_{n_{\mathrm{e}}}[\mathrm{ms}]$ & $\Delta t_{T_{\mathrm{e}}}[\mathrm{ms}]$ \\
\hline \#33 207 & 0.21 & 6.9 & single $f_{\mathrm{ELM}}$ & 93 & 2.5 & 2.5 \\
$\# 33207$ & 0.24 & 11.8 & fast & 286 & & \\
\#33207 & 0.24 & 11.8 & slow & 148 & & \\
\hline \#33194 & 0.37 & 6.9 & single $f_{\mathrm{ELM}}$ & 60 & 2.5 & 7.5 \\
\#33194 & 0.40 & 11.7 & fast & 134 & 2.5 & 3.0 \\
\#33194 & 0.40 & 11.7 & slow & 85 & 2.5 & 3.5 \\
\hline
\end{tabular}

Table 1: ELM cycle types and characteristic pedestal recovery timescales: After a fast, initial recovery phase $n_{\mathrm{e}}$ pedestal $\left(\Delta t_{n_{\mathrm{e}}}\right)$, which duration does not show any dependency on $P_{\text {heat }}$ or $\delta$, the $T_{\mathrm{e}}$ pedestal builds up $\left(\Delta t_{T_{\mathrm{e}}}\right)$. When two $f_{\mathrm{ELM}}$ bands are observed the 'slow' ELM cycle relates to the ELM cycle at a single $f_{\mathrm{ELM}}$.

background, while the lower $f_{\text {ELM }}$ band, the 'slow' ELM cycles have a red background.

In both presented cases of $\delta$, 'slow' and 'fast' ELM cycles appear irregularly, i.e. there is now systematic relation between them. At low $\delta$ it can be seen that the 'fast' ELM cycle ends at reduced pedestal top $p_{\mathrm{e}}$ in comparison to the slow ELM cycle. A summary of the recovery timescales of the analysed discharge intervals is presented in table 1 and compared to the interval with a single $f_{\text {ELM }}$. The $\Delta t_{n_{\mathrm{e}}}$ does not show any dependency on $P_{\text {heat }}$ or $\delta$, while at higher $\delta, \Delta t_{T_{\mathrm{e}}}$ becomes longer, because of the larger ELM loss. When comparing $f_{\mathrm{ELM}}$ of the 'fast' and 'slow' ELM cycle to the discharge intervals with a single $f_{\mathrm{ELM}}$, the 'slow' ELM cycle relates to the ELM cycle observed at a single $f_{\mathrm{ELM}}$, considering that $P_{\text {heat }}$ is higher in the cases with two $f_{\mathrm{ELM}}$ bands. This is further supported by the comparison of the pedestal recovery dynamics of the 'fast' and 'slow' ELM cycles at low and high $\delta$. These are presented in section 5.2 and the detailed pre-ELM pedestal structure of both kinds of ELM cycles is analysed in section 5.3. 

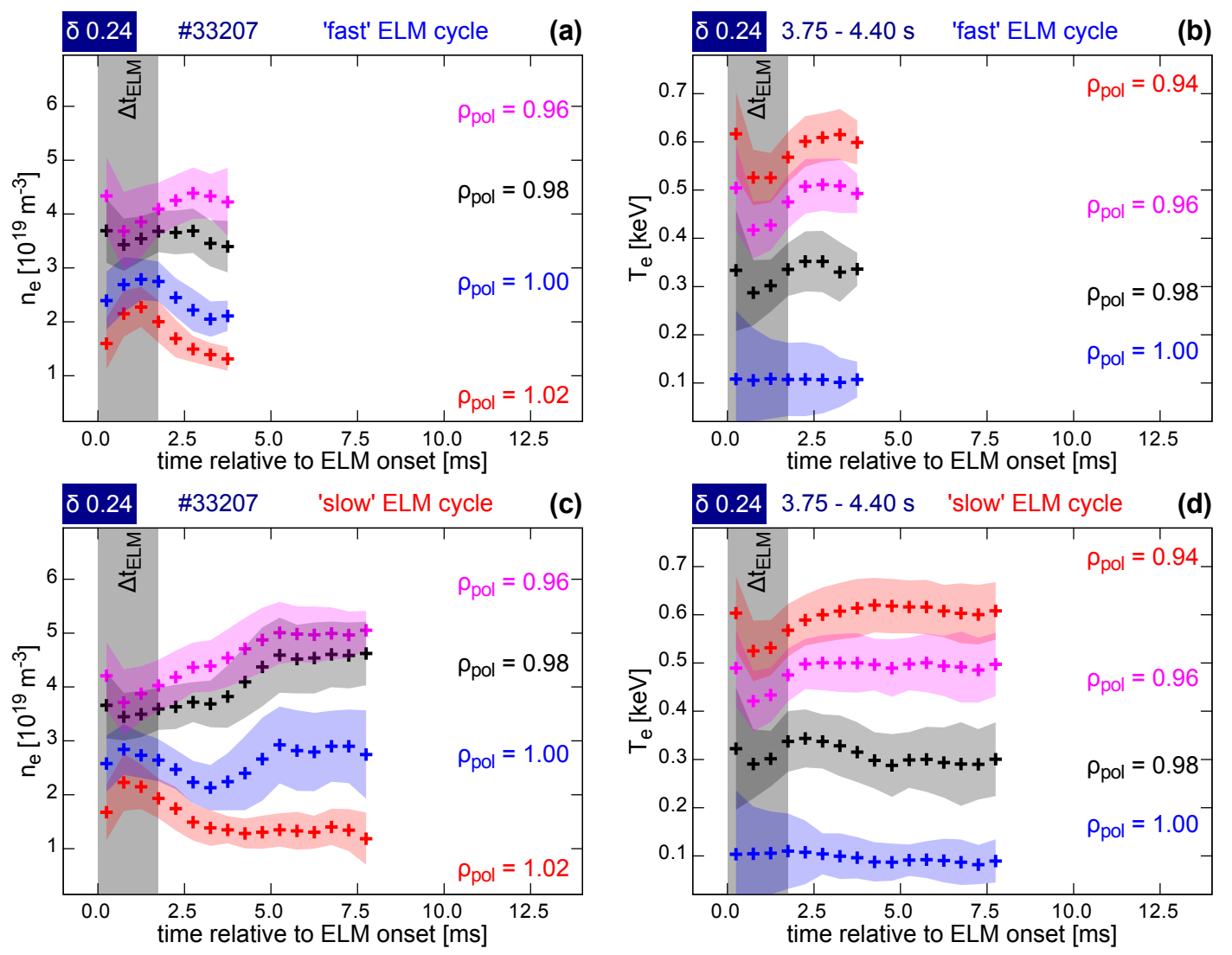

Figure 11: Pedestal evolution at low $\delta$ for the two $f_{\mathrm{ELM}}$ bands: (a,c) $n_{\mathrm{e}}$ and (b,d) $T_{\mathrm{e}}$ at several radial positions $\left(\rho_{\text {pol }}\right)$ for the $(\mathrm{a}, \mathrm{b})$ 'fast' and $(\mathrm{c}, \mathrm{d})$ 'slow' ELM cycles. Roughly $2.0 \mathrm{~ms}$ after the end of the ELM crash $\left(\Delta t_{\mathrm{ELM}}\right)$ the 'fast' ELM cycle is ended by another ELM crash.

\subsection{Pedestal development of 'slow' and 'fast' ELM cycles}

In general, the 'slow' ELM cycles show similar recovery behaviour as the ELM cycles at single $f_{\mathrm{ELM}}$ (see section 4.2). This means that the pedestal evolves through the typical sequence of recovery phases of $n_{\mathrm{e}}, T_{\mathrm{e}}$ and the pre-ELM clamping of the pressure gradient. At $f_{\mathrm{ELM}}>200 \mathrm{~Hz}$ the inter-ELM recovery phases become so short, that a clear separation becomes challenging. In the low $\delta$ case the higher $f_{\mathrm{ELM}}$ is between $250 \mathrm{~Hz}$ and $300 \mathrm{~Hz}$. In combination with the duration of the ELM crash $\left(\Delta t_{\text {ELM }}\right)$ of approximately $1.5 \mathrm{~ms}$, this gives the pedestal only a short time to recover in a 'fast' ELM cycle. In figure 11 the ELM synchronised evolutions of the $n_{\mathrm{e}}$ and $T_{\mathrm{e}}$ pedestal are presented for 'fast' (figures $11 \mathrm{a}$ and $11 \mathrm{~b}$ ) and 'slow' (figures $11 \mathrm{c}$ and $11 \mathrm{~d}$ ) ELM cycles. The $n_{\mathrm{e}}$ and $T_{\mathrm{e}}$ profiles are tracked at equal locations as in figure 9 . In comparison to the 'slow' ELM cycle, the 'fast' ELM cycle immediately ends after a short recovery phase of approximately $2.0 \mathrm{~ms}$. In this case it is not possible to clearly identify the different pedestal recovery phases of $n_{\mathrm{e}}$ and $T_{\mathrm{e}}$ because of the uncertainties of the data (shaded areas). 

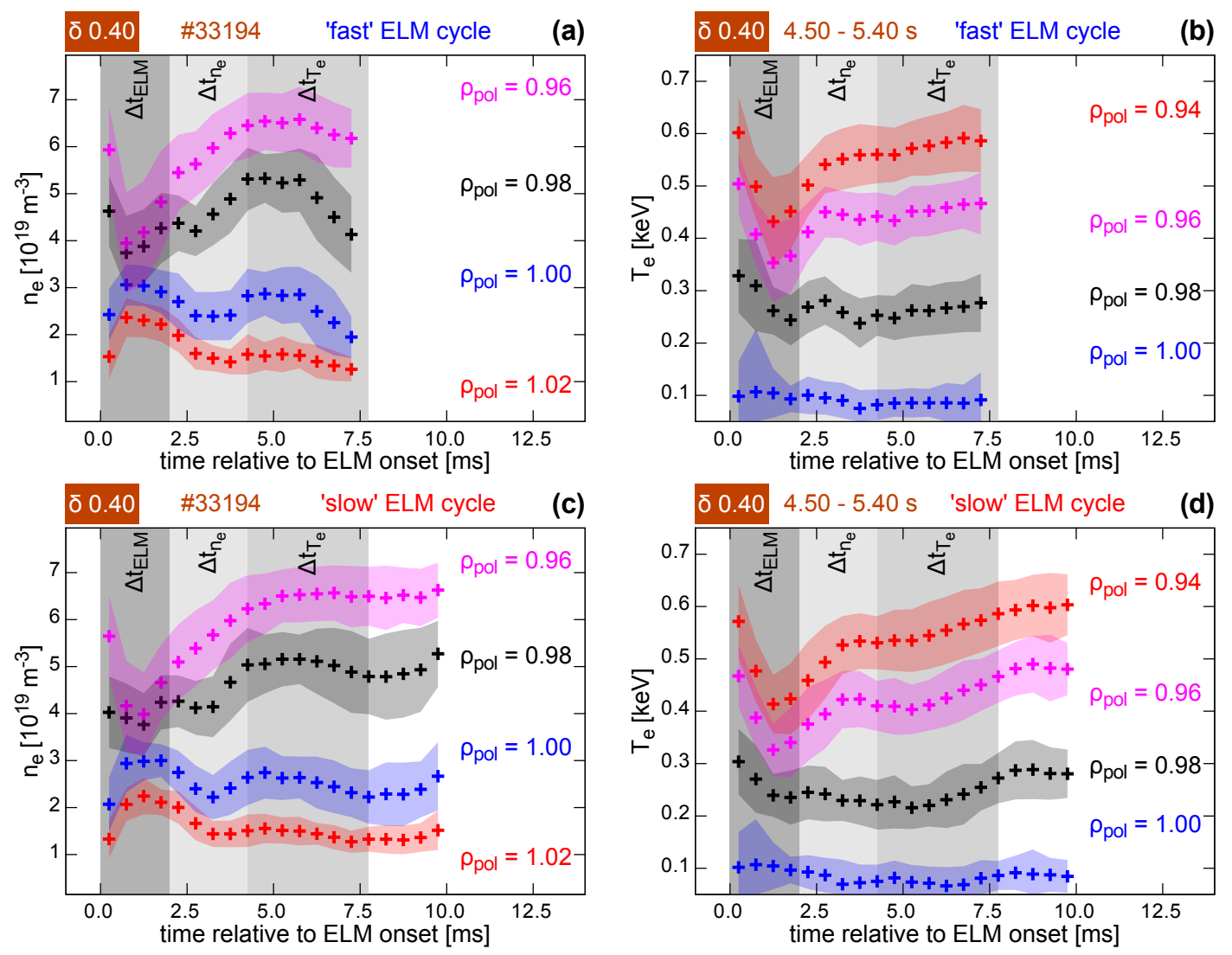

Figure 12: Pedestal evolution at high $\delta$ for the two $f_{\mathrm{ELM}}$ bands: $(\mathrm{a}, \mathrm{c}) n_{\mathrm{e}}$ and $(\mathrm{b}, \mathrm{d}) T_{\mathrm{e}}$ at several radial positions $\left(\rho_{\mathrm{pol}}\right)$ for the $(\mathrm{a}, \mathrm{b})$ 'fast' and $(\mathrm{c}, \mathrm{d})$ 'slow' ELM cycles. At the end of the 'fast' ELM cycle, which appears close to the end of the $T_{\text {e }}$ pedestal recovery phase $\left(\Delta t_{T_{\mathrm{e}}}\right)$, a reduction of the $n_{\mathrm{e}}$ from the separatrix inwards is observed, which is not that pronounced in this phase of the 'slow' ELM cycle.

At higher $\delta$, the limitation in temporal resolution of the inter-ELM phases relaxes since $f_{\mathrm{ELM}}$ is lower. In figure 12 the pedestal development of the high $\delta$ case is shown in a similar setup as in figure 11. Here, the pedestal recovery phases are well resolved and indicated by grey shaded areas. The 'fast' ELM cycle ends towards the end of the $T_{\mathrm{e}}$ pedestal recovery phase and therefore, the pre-ELM phase with clamped pressure gradients is missing. In both types of ELM cycles the $n_{\mathrm{e}}$ and $T_{\mathrm{e}}$ pedestal recovery behaves similar up to the point when the following ELM crash appears. Towards the end of the 'fast' ELM cycle a reduction of the $n_{\mathrm{e}}$ from the separatrix inwards is observed, which corresponds to an inward shift of the $n_{\mathrm{e}}$ profile. This profile movement is not that pronounced in the 'slow' ELM cycle.

The appearance of two distinct $f_{\text {ELM }}$ bands suggests that during the inter-ELM pedestal development, possibly more pedestal configurations, i.e. pedestal structures combined with core conditions, could become unstable, leading to an ELM crash. Changes in $W_{\text {MHD }}$ impact on the PB stability via the Shafranov shift $[50,51,52,53,54]$. To investigate the global $W_{\text {MHD }}$ dynamics in the ELM cycle, the change of $W_{\text {MHD }}$ relative 

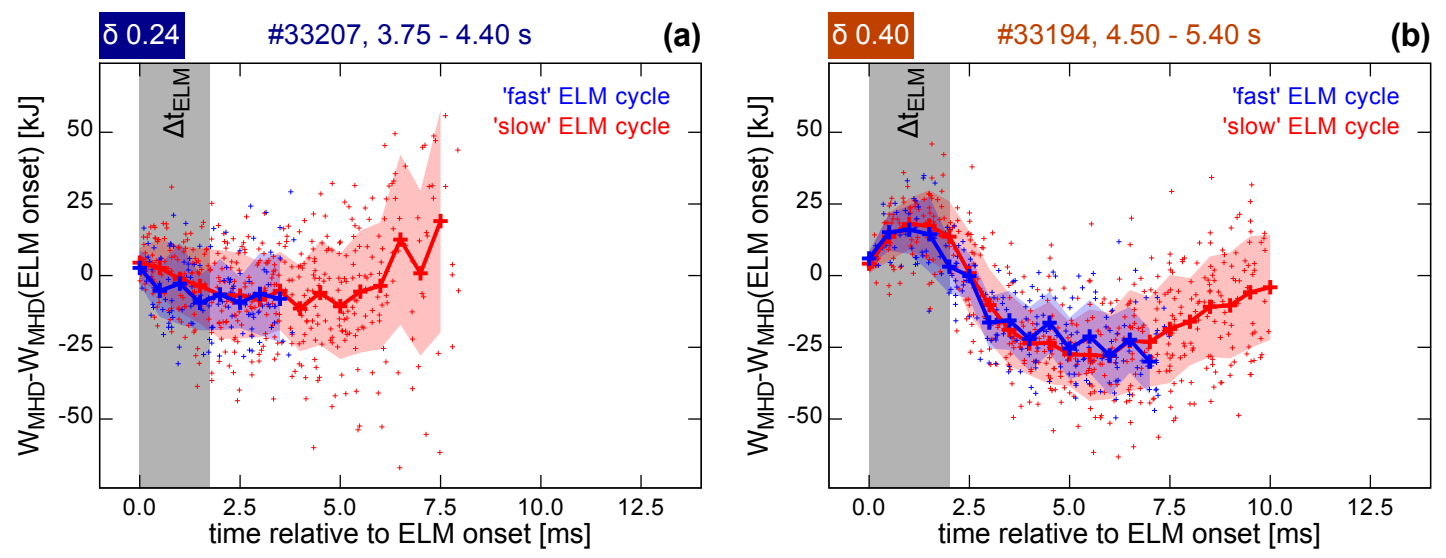

Figure 13: Evolution of $W_{\text {MHD }}$ throughout the ELM cycle: The change of $W_{\text {MHD }}$ relative to $W_{\text {MHD }}$ at the ELM onset for 'fast' (blue) and 'slow' (red) ELM cycle at (a) low and at (b) high $\delta$. The 'fast' ELM cycle ends before the $W_{\text {MHD }}$ increases, which then provides a stabilising effect on the pedestal and extends the 'slow' ELM cycle.

to the $W_{\text {MHD }}$ at the ELM onset for 'fast' and 'slow' ELM cycle are presented in figure 13. The representation of the 'relative $W_{\mathrm{MHD}}$ ' was chosen to emphasise the dynamics of $W_{\mathrm{MHD}}$ and exclude slower trends of $W_{\mathrm{MHD}}$, which evolve on longer time scales than the ELM cycle and therefore, would lead to additional scatter in the data. At high $\delta, W_{\mathrm{MHD}}$ increases in the period of the ELM crash $\left(\Delta t_{\text {ELM }}\right)$, which is not related to any physical reason but rather demonstrates the challenge to reconstruct a plasma equilibrium during a fast, transient phase like an ELM crash. In the inter-ELM period, $W_{\text {MHD }}$ of both types of ELM cycles evolve similarly at low as well as at high $\delta$. It appears that $W_{\text {MHD }}$ in the 'slow' ELM cycle starts to increase exactly at the point when the 'fast' ELM cycle ends. This underlines that the increasing $W_{\text {MHD }}$ helps to stabilise the pedestal and for this reason extends the 'slow' ELM cycle. Therefore, it can be concluded, that at the end of the 'fast' ELM cycle a critical point close to the pedestal stability limit is reached, which can be overcome, when the global $W_{\text {MHD }}$ increases, providing a stabilising effect on the pedestal. To investigate the pedestal stability at the end of both ELM cycles, the pedestal structure is analysed.

\subsection{Pre-ELM pedestal conditions and stability}

Figure 14 compares the pedestal $n_{\mathrm{e}}, T_{\mathrm{e}}$ as well as resulting $p_{\mathrm{e}}$ profiles for 'fast' (blue) and 'slow' (red) ELM cycle. The profiles, represented with solid lines, are averaged in the time interval between $-0.75 \mathrm{~ms}$ and $-0.25 \mathrm{~ms}$ relative to the onset of the following ELM crash, i.e. at the end of the corresponding ELM cycle. The dashed lines indicate the profiles in the 'slow' ELM cycle, in the time interval, when typically the 'fast' ELM cycle ends. The corresponding time intervals are between $3.25 \mathrm{~ms}$ and $3.75 \mathrm{~ms}$ relative to the previous ELM onset at low $\delta$ and between $6.75 \mathrm{~ms}$ and $7.25 \mathrm{~ms}$ relative to the previous ELM onset at high $\delta$. For both cases of $\delta$, the $T_{\mathrm{e}}$ profiles (figures $14 \mathrm{~b}$ and $14 \mathrm{e}$ ) are 

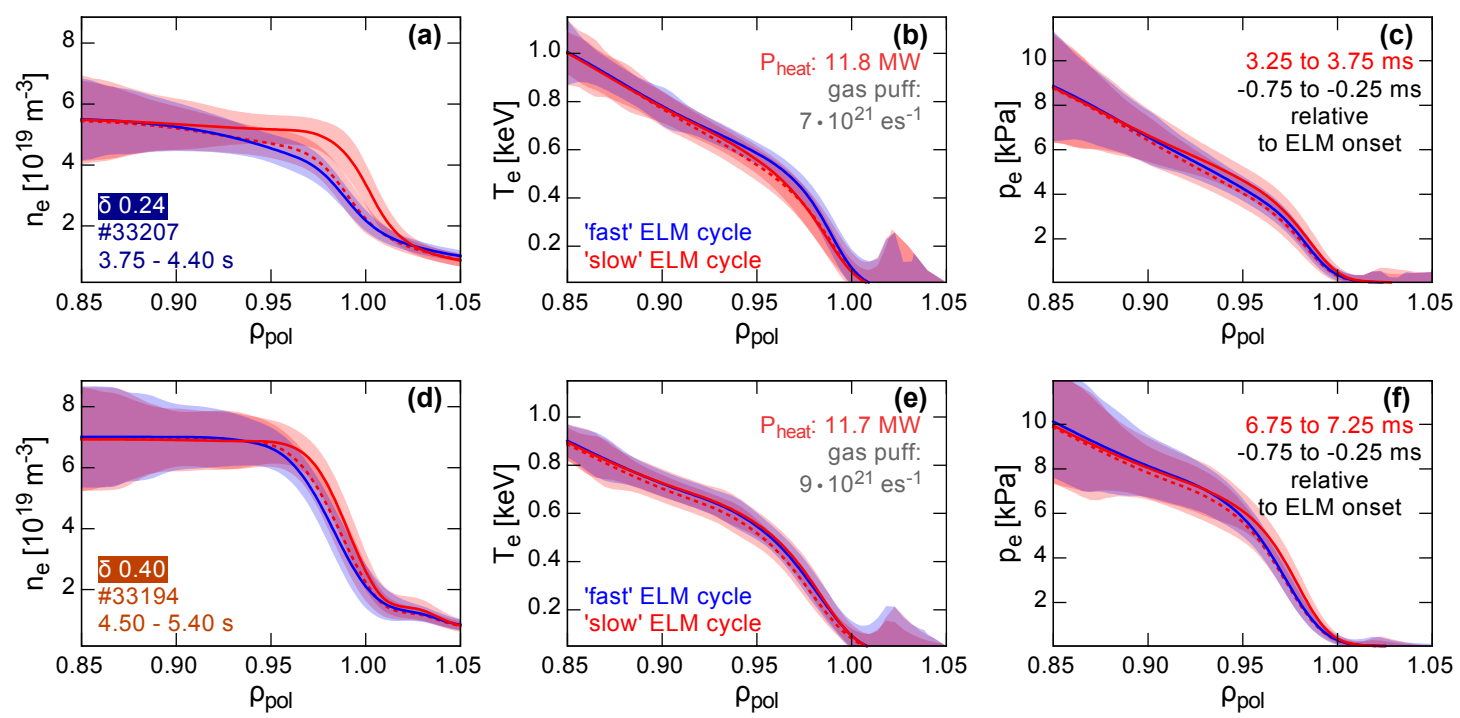

Figure 14: Pre-ELM pedestal profiles at the end of the different ELM cycles: (a,d) $n_{\mathrm{e}}$, (b,e) $T_{\mathrm{e}}$ and $(\mathrm{c}, \mathrm{f}) p_{\mathrm{e}}$ for the $(\mathrm{a}, \mathrm{b}, \mathrm{c})$ low and $(\mathrm{d}, \mathrm{e}, \mathrm{f})$ high $\delta$ cases. The red dashed lines correspond to the profiles in the 'slow' ELM cycle, at the relative time interval relative to the ELM onset, when the 'fast' ELM cycle ends. At the end of the 'fast' ELM cycle the $n_{\mathrm{e}}$ profile (solid blue line) is located further inwards for both $\delta$ in comparison to the end of the 'slow' ELM cycle (solid red line), resulting in a reduced $\nabla p_{\mathrm{e}}$.

similar at the end of the 'fast' and 'slow' ELM cycle. The largest difference is observed in the $n_{\mathrm{e}}$ profiles (figures $14 \mathrm{a}$ and $14 \mathrm{~d}$ ). The pre-ELM $n_{\mathrm{e}}$ profile of the 'fast' ELM cycle is located further inwards and also $\nabla n_{\mathrm{e}}$ is shallower. In the $p_{\mathrm{e}}$ profiles this is reflected by a reduced $\nabla p_{\mathrm{e}}$. The $n_{\mathrm{e}}$ profiles of the 'slow' ELM cycle (red dashed lines) at similar time as the 'fast' ELM cycle ends, are shifted slightly outwards in comparison to the pre-ELM profiles of the 'fast' ELM cycle (blue solid line) and the $T_{\mathrm{e}}$ profiles are located slightly inwards such that the $p_{\mathrm{e}}$ profiles almost overlay. Although, these shifts occur systematically at low and high $\delta$, they are within the experimental uncertainties.

Stability analyses were performed with the corresponding equilibria at the end of the 'fast' and 'slow' ELM cycles. In figure 15 the corresponding $\mathrm{j}$ - $\alpha$ diagrams are presented. At both investigated $\delta$, the operational points at the end of the 'fast' ELM cycles (blue) are located at lower $\alpha_{\max }$ as a result of the inward located $n_{\mathrm{e}}$ profile. The PB stability boundaries are similarly shifted as the operational points. Further, the operational points are close to the PB boundary for both types of ELM cycles, which suggests that it is likely for the pedestal to become unstable.

All in all, the pre-ELM pedestals at the end of the 'fast' and 'slow' ELM cycles agree with PB stability. This can be interpreted such that the pedestal in between ELMs evolves through configurations which become more likely unstable. Therefore, the occurrence of two $f_{\text {ELM }}$ bands is linked to the inter-ELM pedestal stability. The underlying mechanism that leads to this variation of the pedestal stability throughout 

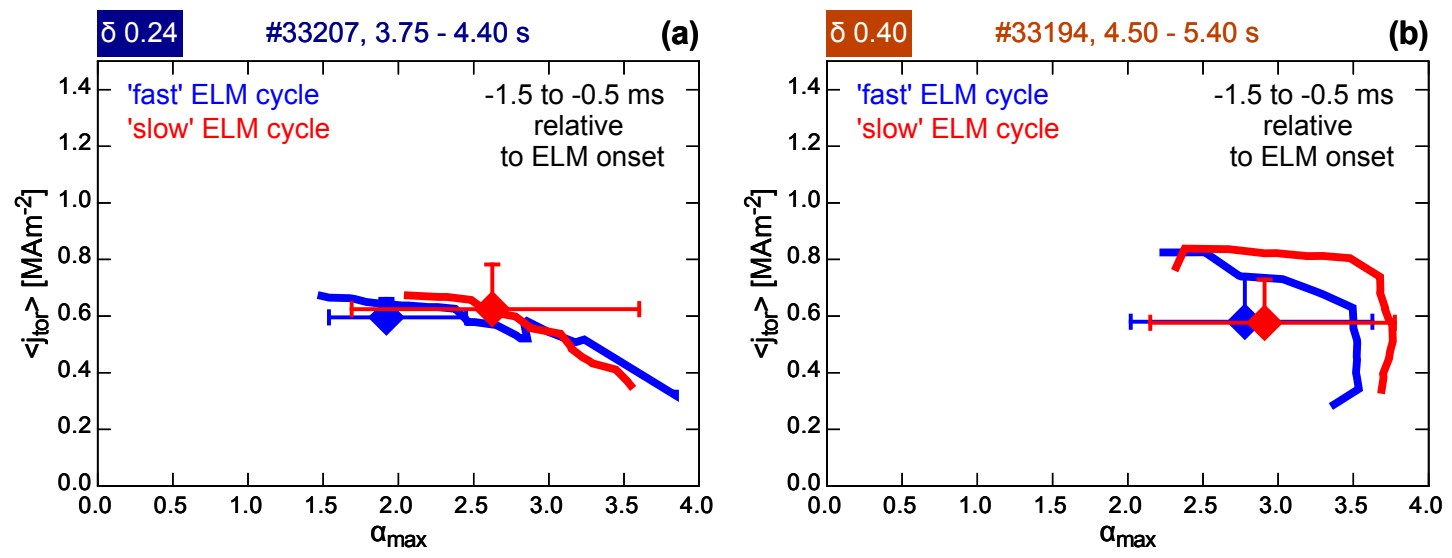

Figure 15: Pre-ELM stability at the end of the 'fast' and 'slow' ELM cycle: (a) at low and (b) high $\delta$. The operational points are located at higher $\alpha_{\max }$ at the end of the 'slow' ELM cycle for both $\delta$ and the PB boundary is shifted correspondingly.

the ELM cycle is the impact of $W_{\mathrm{MHD}}$. Since the inter-ELM evolution of $W_{\mathrm{MHD}}$ is not rigidly coupled to the evolution of the pedestal pressure, there is some degree of freedom which allows the formation of two separated $f_{\text {ELM }}$ bands. The 'fast' ELM cycle ends, i.e. an ELM crash appears, at a point of relatively low $W_{\text {MHD }}$. When this point is overcome in the slow ELM cycle, $W_{\mathrm{MHD}}$, respectively the Shafranov shift increases, stabilising the pedestal and allowing for its further evolution.

\section{Discussion of the main observations and conclusions}

The presented results of this work are consistent with previous studies on the impact of $\delta$ on the pedestal. It extends these results towards a deeper analysis of the inter-ELM pedestal evolution. A similar sequence of the pedestal recovery phases is identified for all $\delta$. First, the $n_{\mathrm{e}}$ pedestal is immediately established after the ELM crash, then the $T_{\mathrm{e}}$ pedestal builds up and after that a period with clamped pressure gradient appears. The decrease of $f_{\mathrm{ELM}}$ with increasing $\delta$ is caused by prolongation of the last two inter-ELM pedestal recovery phases. For the $T_{\mathrm{e}}$ pedestal recovery phase, the prolongation is related to the higher ELM loss, since at a constant recovery rate a larger drop in $T_{\mathrm{e}}$ needs longer to recover. Further, the pre-ELM phase with clamped pressure gradient is significantly extended at high $\delta$. This could be either caused by an increased particle and heat flux across the edge or by the beneficial effect of $\delta$ on the edge stability.

Higher $\delta$ enhances the pedestal top $n_{\mathrm{e}}$ although $W_{\mathrm{MHD}}$ is not strongly affected in the presented experiments, which might be partially related to increased radiation. The pre-ELM pedestal structure agrees with $\mathrm{PB}$ theory and at higher $\delta$ the $\mathrm{PB}$ stability boundary is shifted towards larger $\left\langle j_{\text {tor }}\right\rangle$ and $\alpha_{\max }$ allowing steeper pedestal pressure profiles and higher pedestal top pressures. The enhanced pedestal top $n_{\mathrm{e}}$ at higher $\delta$ is already established during the initial recovery phase of the $n_{\mathrm{e}}$ pedestal, immediately after an ELM crash. This indicates that the $n_{\mathrm{e}}$ pedestal structure has an important impact 
on the $p_{\mathrm{e}}$ profile, which is set by the PB stability, and the $T_{\mathrm{e}}$ pedestal contributes in between those 'boundaries' due to its slower recovery timescale. However, this simple picture does not imply that the temperature has a minor role in forming the pedestal. Throughout this work the evolution of the electron contribution to the pedestal was investigated, however, the ion temperature $\left(T_{\mathrm{i}}\right)$ can be decoupled from $T_{\mathrm{e}}$. Especially, the $T_{\mathrm{i}}$ pedestal exhibits a faster evolution throughout the ELM cycle, which is coupled to the recovery timescale of the $n_{\mathrm{e}}$ pedestal and therefore, faster than the recovery of the $T_{\mathrm{e}}$ pedestal [55]. This becomes relevant, when the temporal dynamics of the PB stability throughout the ELM cycle is analysed since the total pressure as well as the edge current can be affected. With respect to the presented work, the different dynamics of the $T_{\mathrm{i}}$ play a minor role, since the stability was only analysed for a single time slice relative to the ELM onset. $T_{\mathrm{i}}$ measurements with low temporal resolution were used to provide information on the ion contribution the total pressure, which is sufficient for the 'static' stability analysis. To resolve the full dynamics of PB stability throughout the ELM cycle, the $T_{\mathrm{i}}$ profile dynamics need to be taken into account. For this reason, future studies using measurements with high temporally resolved $T_{\mathrm{i}}$ profiles will need to address the main ion contribution to the pressure in the $n_{\mathrm{e}}$ pedestal recovery phase for varying $\delta$.

The occurrence of two $f_{\text {ELM }}$ bands has been shown to be connected to the interELM pedestal stability. Both types of ELM cycles evolve through a similar pedestal development. It is important to notice, that the simple picture of a temporally localised stability boundary in $\mathrm{j}-\alpha$ space is incomplete. In addition to the operational point of the plasma equilibrium, which moves according to the evolution of $\alpha_{\max }$ and $\left\langle j_{\text {tor }}\right\rangle$ [56], also the stability boundary changes correspondingly [57]. This can be explained by the evolution of the global plasma parameters such as $W_{\mathrm{MHD}}$. Since the evolution of $W_{\mathrm{MHD}}$ is not rigidly coupled to the pedestal pressure, phases in the ELM cycle can appear where $W_{\text {MHD }}$ is too low to stabilise the pedestal pressure gradient. For this reason, it is possible that, throughout the inter-ELM evolution of the pedestal, certain pedestal configurations become more likely unstable.

At the end of a 'fast' ELM cycle the $n_{\mathrm{e}}$ profile is located radially inwards relative to the $n_{\mathrm{e}}$ profile at the end of a 'slow' ELM cycle. At first glance, this might oppose previous studies, where radially inwards shifted $n_{\mathrm{e}}$ profiles tend to lead to more stable conditions $[16,58]$. But in the presented cases the shift appears during the dynamics of an ELM cycle, at a reduced $W_{\mathrm{MHD}}$ in comparison to the end of the 'slow' ELM cycle i.e. the global plasma conditions at the end of 'fast' and 'slow' ELM cycle differ too. The performed PB stability calculations point into a similar direction. Disregarding the large experimental uncertainties, the operational point and stability boundary tend to be located at lower $\mathrm{j}-\alpha$ at the end of the 'fast' ELM cycle. Under such a combination of conditions, which are close to the PB stability limit, ELM crashes are more likely to occur. However, when the unstable situation, which appears at the end of the 'fast' ELM cycle, can be overcome, $W_{\mathrm{MHD}}$ can evolve further up to the point, where another $\mathrm{PB}$ unstable configuration is reached, which marks the end of the 'slow' ELM cycle. 
In summary, the key results of the presented work point out that the sequence of inter-ELM pedestal recovery is not affected by plasma shaping for the examined range. The pedestal top density, which exhibits a dependency on the shaping, is already established in the initial pedestal recovery phase. The appearance of two distinct $f_{\mathrm{ELM}}$ bands is associated to the not rigidly coupled evolution of the pedestal and the global plasma. To fully understand and model the interaction of core and edge dynamics, especially, the different timescales of their evolution as well as their coupling need to be considered.

\section{Acknowledgments}

The fruitful discussions with H. Meyer and P. A. Schneider as well as their helpful comments to the conducted work are warmly acknowledged.

This work has been carried out within the framework of the EUROfusion Consortium and has received funding from the Euratom research and training programme 2014-2018 under grant agreement No 633053. The views and opinions expressed herein do not necessarily reflect those of the European Commission.

\section{References}

[1] Janeschitz G, JCT I and HTs 2001 Journal of Nuclear Materials 290 1-11 URL http://dx.doi. org/10.1016/S0022-3115(00)00623-1

[2] Loarte A, Saibene G, Sartori R, Campbell D, Becoulet M, Horton L, Eich T, Herrmann A, Matthews G, Asakura N, Chankin A, Leonard A, Porter G, Federici G, Janeschitz G, Shimada M and Sugihara M 2003 Plasma Physics and Controlled Fusion 45 1549-1569 URL http: //dx.doi.org/10.1088/0741-3335/45/9/302

[3] Wenninger R P, Bernert M, Eich T, Fable E, Federici G, Kallenbach A, Loarte A, Lowry C, McDonald D, Neu R, Putterich T, Schneider P, Sieglin B, Strohmayer G, Reimold F and Wischmeier M 2014 Nuclear Fusion 54114003 URL http://dx.doi.org/10.1088/0029-5515/ $54 / 11 / 114003$

[4] Zohm H 2014 Magnetohydrodynamic Stability of Tokamaks (Weinheim: WILEY-VCH)

[5] Snyder P B, Wilson H R, Osborne T H and Leonard A W 2004 Plasma Physics and Controlled Fusion 46 A131-A141 URL http://dx.doi.org/10.1088/0741-3335/46/5a/014

[6] Pankin A Y, Rafiq T, Kritz A H, Park G Y, Snyder P B and Chang C S 2017 Physics of Plasmas 24062502 URL http://dx.doi.org/10.1063/1.4984256

[7] Saibene G, Horton L D, Sartori R, Balet B, Clement S, Conway G D, Cordey J G, Esch H P L D, Ingesson L C, Lingertat J, Monk R D, Parail V V, Smith R J, Taroni A, Thomsen K and von Hellermann M G 1999 Nuclear Fusion 39 1133-1156 URL http://dx.doi.org/Doi10.1088/ $0029-5515 / 39 / 9 / 307$

[8] Suttrop W, Gruber O, Kurzan B, Murmann H D, Neuhauser J, Schweinzer J, Stober J, Treutterer W and Team A U 2000 Plasma Physics and Controlled Fusion 42 A97-A102 URL http: //dx.doi.org/10.1088/0741-3335/42/5a/308

[9] Osborne T H, Ferron J R, Groebner R J, Lao L L, Leonard A W, Mahdavi M A, Maingi R, Miller R L, Turnbull A D, Wade M and Watkins J 2000 Plasma Physics and Controlled Fusion 42 A175-A184 URL http://dx.doi.org/10.1088/0741-3335/42/5a/319

[10] Kallenbach A, Beurskens M N A, Korotkov A, Lomas P, Suttrop W, Charlet M, McDonald D C, 
Milani F, Rapp J, Stamp M, contributors E J W and Team A U 2002 Nuclear Fusion 42 1184-1192 URL http://dx.doi.org/10.1088/0029-5515/42/10/302

[11] Saibene G, Sartori R, Loarte A, Campbell D J, Lomas P J, Parail V, Zastrow K D, Andrew Y, Sharapov S, Korotkov A, Becoulet M, Huysmans G T A, Koslowski H R, Budny R, Conway G D, Stober J, Suttrop W, Kallenbach A, von Hellermann M and Beurskens M 2002 Plasma Physics and Controlled Fusion 44 1769-1799 URL http://dx.doi.org/10.1088/0741-3335/44/9/301

[12] Suttrop W 2000 Plasma Physics and Controlled Fusion 42 A1-A14 URL http://dx.doi.org/ $10.1088 / 0741-3335 / 42 / 5 a / 301$

[13] Jackson G, Murakami M, McKee G, Baker D, Boedo J, Haye R L, Lasnier C, Leonard A, Messiaen A, Ongena J, Staebler G, Unterberg B, Wade M, Watkins J and West W 2002 Nuclear Fusion 4228 URL http://dx.doi.org/10.1088/0029-5515/42/1/305

[14] Beurskens M N A, Arnoux G, Brezinsek A S, Challis C D, de Vries P C, Giroud C, Huber A, Jachmich S, McCormick K, Pitts R A, Rimini F G, Alfier A, de la Luna E, Fundamenski W, Gerasimov S, Giovannozzi E, Joffrin E, Kempenaars M, Litaudon X, Loarer T, Lomas P, Mailloux J, Pasqualotto R, Pericoli-Ridolfini V, Pugno R, Rachlew E, Saarelma S, Solano E, Walsh M, Zabeo L, Zastrow K D and Contributors J E 2008 Nuclear Fusion 48095004 URL http://dx.doi.org/10.1088/0029-5515/48/9/095004

[15] Schneider P A, Wolfrum E, Dunne M G, Dux R, Gude A, Kurzan B, Putterich T, Rathgeber S K, Vicente J, Weller A, Wenninger R and Team A U 2014 Plasma Physics and Controlled Fusion 56 025011 URL http://dx.doi.org/10.1088/0741-3335/56/2/025011

[16] Dunne M G, Potzel S, Reimold F, Wischmeier M, Wolfrum E, Frassinetti L, Beurskens M, Bilkova P, Cavedon M, Fischer R, Kurzan B, Laggner F M, McDermott R M, Tardini G, Trier E, Viezzer E and Willensdorfer M 2017 Plasma Physics and Controlled Fusion 59014017 URL http://dx.doi.org/10.1088/0741-3335/59/1/014017

[17] Frassinetti L, Dunne M G, Beurskens M, Wolfrum E, Bogomolov A, Carralero D, Cavedon M, Fischer R, Laggner F M, McDermott R M, Meyer H, Tardini G and Viezzer E 2017 Nuclear Fusion 57022004 URL http://dx.doi.org/10.1088/0029-5515/57/2/022004

[18] Urano H, Suttrop W, Horton L D, Herrmann A, Fuchs J C and Team A U 2003 Plasma Physics and Controlled Fusion 45 1571-1596 URL http://dx.doi.org/10.1088/0741-3335/45/9/303

[19] Beurskens M N A, Schweinzer J, Angioni C, Burckhart A, Challis C D, Chapman I, Fischer R, Flanagan J, Frassinetti L, Giroud C, Hobirk J, Joffrin E, Kallenbach A, Kempenaars M, Leyland M, Lomas P, Maddison G, Maslov M, McDermott R, Neu R, Nunes I, Osborne T, Ryter F, Saarelma S, Schneider P A, Snyder P, Tardini G, Viezzer E and Wolfrum E 2013 Plasma Physics and Controlled Fusion 55 URL http://dx.doi.org/10.1088/0741-3335/55/12/124043

[20] Leyland M J, Beurskens M N A, Frassinetti L, Osborne T, Snyder P B, Giroud C, Jachmich S, Maddison G, Lomas P, von Thun C P, Saarelma S, Saibene G, Gibson K J and Collaborators J E 2013 Nuclear Fusion 53 URL http://dx.doi.org/10.1088/0029-5515/53/8/083028

[21] Lennholm M, Beaumont P S, Carvalho I S, Chapman I T, Felton R, Frigione D, Garzotti L, Goodyear A, Graves J, Grist D, Jachmich S, Lang P, Lerche E, de la Luna E, Mooney R, Morris J, Nave M F F, Rimini F, Sips G, Solano E, Tsalas M and Contributors J E 2015 Nuclear Fusion 55063004 URL http://dx.doi.org/10.1088/0029-5515/55/6/063004

[22] Giroud C, Jachmich S, Jacquet P, Jarvinen A, Lerche E, Rimini F, Aho-Mantila L, Aiba N, Balboa I, Belo P, Angioni C, Beurskens M, Brezinsek S, Casson F J, Coffey I, Cunningham G, Delabie E, Devaux S, Drewelow P, Frassinetti L, Figueiredo A, Huber A, Hillesheim J, Garzotti L, Goniche M, Groth M, Kim H T, Leyland M, Lomas P, Maddison G, Marsen S, Matthews G, Meigs A, Menmuir S, Puetterich T, van Rooij G, Saarelma S, Stamp M, Urano H, Webster A and Contributors J E 2015 Plasma Physics and Controlled Fusion 57 URL http://dx.doi.org/10.1088/0741-3335/57/3/035004

[23] Schneider P A, Orte L B, Burckhart A, Dunne M G, Fuchs C, Gude A, Kurzan B, Suttrop W, Wolfrum E and Team A U 2015 Plasma Physics and Controlled Fusion 57014029 URL http://dx.doi.org/10.1088/0741-3335/57/1/014029 
[24] Burckhart A, Wolfrum E, Fischer R, Lackner K, Zohm H and Team A U 2010 Plasma Physics and Controlled Fusion 52105010 URL http://dx.doi.org/10.1088/0741-3335/52/10/105010

[25] Burckhart A, Dunne M, Wolfrum E, Fischer R, McDermott R, Viezzer E and Willensdorfer M 2016 Nuclear Fusion 56056011 URL http://dx.doi.org/10.1088/0029-5515/56/5/056011

[26] Ahn J W, Kim H S, Park Y S, Terzolo L, Ko W H, Park J K, England A C, Yoon S W, Jeon Y M, Sabbagh S A, Bae Y S, Bak J G, Hahn S H, Hillis D L, Kim J, Kim W C, Kwak J G, Lee K D, Na Y S, Nam Y U, Oh Y K and Park S I 2012 Nuclear Fusion 52114001 URL http://dx.doi.org/10.1088/0029-5515/52/11/114001

[27] Degeling A W, Martin Y R, Bak P E, Lister J B and Llobet V 2001 Plasma Physics and Controlled Fusion 43 1671-1698 URL http://dx.doi.org/10.1088/0741-3335/43/12/304

[28] Laggner F M, Wolfrum E, Cavedon M, Mink F, Viezzer E, Dunne M G, Manz P, Doerk H, Birkenmeier G, Fischer R, Fietz S, Maraschek M, Willensdorfer M and Aumayr F 2016 Plasma Physics and Controlled Fusion 58065005 URL http://dx.doi.org/10.1088/0741-3335/58/ $6 / 065005$

[29] Ryter F, Rathgeber S K, Orte L B, Bernert M, Conway G D, Fischer R, Happel T, Kurzan B, McDermott R M, Scarabosio A, Suttrop W, Viezzer E, Willensdorfer M, Wolfrum E and Team A U 2013 Nuclear Fusion 53113003 URL http://dx.doi.org/10.1088/0029-5515/53/11/ 113003

[30] Fischer R, Fuchs C J, Kurzan B, Suttrop W, Wolfrum E and Team A U 2010 Fusion Science and Technology 58 675-684 URL http://dx.doi.org/10.13182/FST10-110

[31] Mlynek A, Reich M, Giannone L, Treutterer W, Behler K, Blank H, Buhler A, Cole R, Eixenberger H, Fischer R, Lohs A, Luddecke K, Merkel R, Neu G, Ryter F, Zasche D and Team A U 2011 Nuclear Fusion 51043002 URL http://dx.doi.org/10.1088/0029-5515/51/4/043002

[32] Willensdorfer M, Birkenmeier G, Fischer R, Laggner F M, Wolfrum E, Veres G, Aumayr F, Carralero D, Guimarais L, Kurzan B and Team A U 2014 Plasma Physics and Controlled Fusion 56 025008 URL http://dx.doi.org/10.1088/0741-3335/56/2/025008

[33] Willensdorfer M, Denk S S, Strumberger E, Suttrop W, Vanovac B, Brida D, Cavedon M, Classen I, Dunne M, Fietz S, Fischer R, Kirk A, Laggner F M, Liu Y Q, Odstril T, Ryan D A, Viezzer E, Zohm H and Luhmann I C 2016 Plasma Physics and Controlled Fusion 58114004 URL http://dx.doi.org/10.1088/0741-3335/58/11/114004

[34] Rathgeber S K, Barrera L, Eich T, Fischer R, Nold B, Suttrop W, Willensdorfer M, Wolfrum E and Team A U 2013 Plasma Physics and Controlled Fusion 55025004 URL http://dx.doi. org/10.1088/0741-3335/55/2/025004

[35] Kurzan B, Murmann H, Salzmann H and Team A U 2001 Review of Scientific Instruments 72 1111-1114 URL http://dx.doi.org/10.1063/1.1321747

[36] Neuhauser J, Coster D, Fahrbach H U, Fuchs J C, Haas G, Herrmann A, Horton L, Jakobi M, Kallenbach A, Laux M, Kim J W, Kurzan B, Muller H W, Murmann H, Neu R, Rohde V, Sandmann W, Suttrop W, Wolfrum E and Team A U 2002 Plasma Physics and Controlled Fusion 44 855-869 URL http://dx.doi.org/10.1088/0741-3335/44/6/316

[37] Fischer R, Bock A, Dunne M, Fuchs J C, Giannone L, Lackner K, McCarthy P J, Poli E, Preuss R, Rampp M, Schubert M, Stober J, Suttrop W, Tardini G, Weiland M and Team A U 2016 Fusion Science and Technology 69 526-536 URL http://dx.doi.org/10.13182/fst15-185

[38] Beurskens M N A, Dunne M G, Frassinetti L, Bernert M, Cavedon M, Fischer R, Jrvinen A, Kallenbach A, Laggner F M, McDermott R M, Potzel S, Schweinzer J, Tardini G, Viezzer E, Wolfrum E, Team A U and Team E M 2016 Nuclear Fusion 56056014 URL http: //dx.doi.org/10.1088/0029-5515/56/5/056014

[39] Stober J, Gruber O, Kallenbach A, Mertens V, Ryter F, Stabler A, Suttrop W, Treutterer W and Team A U 2000 Plasma Physics and Controlled Fusion 42 A211-A216 URL http: //dx.doi.org/10.1088/0741-3335/42/5a/324

[40] Maggi C F, Saarelma S, Casson F J, Challis C, de la Luna E, Frassinetti L, Giroud C, Joffrin E, Simpson J, Beurskens M, Chapman I, Hobirk J, Leyland M, Lomas P, Lowry 
C, Nunes I, Rimini F, Sips A C C and Urano H 2015 Nuclear Fusion 55113031 URL http://dx.doi.org/10.1088/0029-5515/55/11/113031

[41] Kallenbach A, Dux R, Mayer M, Neu R, Putterich T, Bobkov V, Fuchs J C, Eich T, Giannone L, Gruber O, Herrmann A, Horton L D, Maggi C F, Meister H, Muller H W, Rohde V, Sips A, Stabler A, Stober J and Team A U 2009 Nuclear Fusion 49 URL http://dx.doi.org/10. 1088/0029-5515/49/4/045007

[42] Dux R, Neu R, Peeters A G, Pereverzev G, Muck A, Ryter F, Stober J and Team A U 2003 Plasma Physics and Controlled Fusion 45 1815-1825 URL http://dx.doi.org/10.1088/0741-3335/ $45 / 9 / 317$

[43] Challis C D, Garcia J, Beurskens M, Buratti P, Delabie E, Drewelow P, Frassinetti L, Giroud C, Hawkes N, Hobirk J, Joffrin E, Keeling D, King D B, Maggi C F, Mailloux J, Marchetto C, McDonald D, Nunes I, Pucella G, Saarelma S and Simpson J 2015 Nuclear Fusion 55053031 URL http://dx.doi.org/10.1088/0029-5515/55/5/053031

[44] Snyder P B, Wilson H R, Ferron J R, Lao L L, Leonard A W, Osborne T H, Turnbull A D, Mossessian D, Murakami M and Xu X Q 2002 Physics of Plasmas 9 2037-2043 URL http://dx.doi.org/10.1063/1.1449463

[45] Huysmans G T A 2005 Plasma Physics and Controlled Fusion 47 B165-B178 URL http: //dx.doi.org/10.1088/0741-3335/47/12b/S13

[46] Dunne M G, Frassinetti L, Beurskens M N A, Cavedon M, Fietz S, Fischer R, Giannone L, Huijsmans G T A, Kurzan B, Laggner F, McCarthy P J, McDermott R M, Tardini G, Viezzer E, Willensdorfer M, Wolfrum E, Team E M and Team A U 2017 Plasma Physics and Controlled Fusion 59 025010 URL http://dx.doi.org/10.1088/1361-6587/59/2/025010

[47] Maggi C F, Frassinetti L, Horvath L, Lunniss A, Saarelma S, Wilson H, Flanagan J, Leyland M, Lupelli I, Pamela S, Urano H, Garzotti L, Lerche E, Nunes I, Rimini F and Contributors J 2017 Nuclear Fusion 57 URL http://dx.doi.org/10.1088/1741-4326/aa7e8e

[48] Laggner F M, Wolfrum E, Cavedon M, Mink F, Bernert M, Dunne M G, Schneider P A, Kappatou A, Birkenmeier G, Fischer R, Willensdorfer M, Aumayr F, the EUROfusion MST1 team and the ASDEX Upgrade team 2017 Physics of Plasmas 24056105 URL http://dx.doi.org/10. 1063/1.4977461

[49] Mink F, Wolfrum E, Maraschek M, Zohm H, Horvth L, Laggner F M, Manz P, Viezzer E and Stroth U 2016 Plasma Physics and Controlled Fusion 58125013 URL http://dx.doi.org/10. 1088/0741-3335/58/12/125013

[50] Snyder P B, Burrell K H, Wilson H R, Chu M S, Fenstermacher M E, Leonard A W, Moyer R A, Osborne T H, Umansky M, West W P and Xu X Q 2007 Nuclear Fusion 47 961-968 URL http://dx.doi.org/10.1088/0029-5515/47/8/030

[51] Snyder P B, Aiba N, Beurskens M, Groebner R J, Horton L D, Hubbard A E, Hughes J W, Huysmans G T A, Kamada Y, Kirk A, Konz C, Leonard A W, Lonnroth J, Maggi C F, Maingi R, Osborne T H, Oyama N, Pankin A, Saarelma S, Saibene G, Terry J L, Urano H and Wilson H R 2009 Nuclear Fusion 49 085035 URL http://dx.doi.org/10.1088/0029-5515/49/8/085035

[52] Chapman I T, Simpson J, Saarelma S, Kirk A, O'Gorman T, Scannell R and Team M 2015 Nuclear Fusion 55013004 URL http://dx.doi.org/Artn01300410.1088/0029-5515/55/1/013004

[53] Connor J W, Ham C J and Hastie R J 2016 Plasma Physics and Controlled Fusion 58085002 URL http://dx.doi.org/10.1088/0741-3335/58/8/085002

[54] Urano H, Saarelma S, Frassinetti L, Aiba N, Maggi C F, Chapman I T, Lupelli I, Challis C, Leyland M, Beurskens M, Kamiya K, Giroud C, Pamela S, Team J and Contributors J 2016 Global stabilization effect of shafranov shift on the edge pedestal plasmas in jet and jt-60u URL https://nucleus.iaea.org/sites/fusionportal/Shared\%20Documents/FEC\%202016/ fec2016-preprints/preprint0296.pdf\#search=urano

[55] Cavedon M, Ptterich T, Viezzer E, Laggner F M, Burckhart A, Dunne M, Fischer R, Lebschy A, Mink F, Stroth U, Willensdorfer M and Wolfrum E 2017 Plasma Physics and Controlled Fusion 59105007 URL http://dx.doi.org/10.1088/1361-6587/aa7ad0 
[56] Dickinson D, Saarelma S, Scannell R, Kirk A, Roach C M and Wilson H R 2011 Plasma Physics and Controlled Fusion 53115010 URL http://dx.doi.org/10.1088/0741-3335/53/11/115010

[57] Wolfrum E, Viezzer E, Burckhart A, Dunne M G, Schneider P A, Willensdorfer M, Fable E, Fischer R, Hatch D, Jenko F, Kurzan B, Manz P, Rathgeber S K and Team A U 2015 Nuclear Fusion 55053017 URL http://dx.doi.org/10.1088/0029-5515/55/5/053017

[58] Stefanikova E, Frassinetti L, Saarelma S, Loarte A, Nunes I, Lomas P, Rimini F, Drewelow P, Garzotti L and Kruezi U 2016 Effect of the relative shift between the electron density and temperature pedestal position on the pedestal stability in jet-ilw Proc. 43rd European Physical Society Conf. on Plasma Physics (EPS) vol 40 URL http://ocs . ciemat.es/EPS2016PAP/pdf/ 04.117.pdf 